\title{
Surgery along star-shaped plumbings and exotic smooth structures on 4-manifolds
}

\author{
ÇAĞRI KARAKURT \\ LAURA STARKSTON
}

\begin{abstract}
We define a new 4-dimensional symplectic cut and paste operation which is analogous to Fintushel and Stern's rational blow-down. We use this operation to produce multiple constructions of symplectic smoothly exotic complex projective spaces blown up eight, seven, and six times. We also show how this operation can be used in conjunction with knot surgery to construct an infinite family of minimal exotic smooth structures on the complex projective space blown-up seven times.
\end{abstract}

53Dxx; 57R57

\section{Introduction}

Fintushel and Stern [14] introduced a cut and paste operation for 4-manifolds called rational blow-down. They used it to compute the Donaldson polynomial of the logarithmic transforms of the elliptic surfaces. Since then, the rational blow-down operation has proven to be very useful in 4-dimensional topology. It was a useful constructional tool in the exotic copies of blown-up complex projective plane; see Park [36], Park, Stipsicz and Szabó [37], Stipsicz and Szabó [42], Fintushel and Stern [16], and Michalogiorgaki [30]. It can be used to construct symplectic manifolds, as shown by Symington [44; 45] and Gay and Stipsicz [22], and in the presence of a certain Lefschetz fibration structure one can reinterpret it as a monodromy substitution; see Endo and Gurtas [10] and Endo, Mark and Van Horn-Morris [11].

The purpose of the present paper is to define a new cut and paste operation, called star surgery, which is a strong generalization of Fintushel and Stern's rational blow-down. Just like rational blow-down, our operation reduces $b_{2}^{-}$of the manifold to which it is applied. Moreover it can be performed symplectically and can be seen as a monodromy substitution.

The original rational blow-down operation, and its generalizations - Park [35], Stipsicz, Szabó and Wahl [43], Bhupal and Stipsicz [7] — amount to removing the neighborhood of a union of spheres which intersect according to a particular plumbing tree and 
regluing a rational ball which has the same boundary as the neighborhood. Our star surgery operation is similarly defined. First identify (symplectic) spheres which intersect according to a star-shaped graph with a negativity condition on the central vertex. The star surgery operation cuts out a neighborhood of these spheres and replaces it by an alternate symplectic filling of the induced contact boundary. It is shown by the second author [40] that these alternate fillings always have smaller Euler characteristic than the neighborhood of spheres, and are negative-definite. Unlike the rational blowdown we do not require the alternate filling to be a rational homology ball. This greatly generalizes the set of configurations of spheres which we can consider for these operations.

By reinterpreting this operation as a monodromy substitution, one can show that some star surgeries are obtained by a sequence of rational blow-downs. The spheres to rationally blow down after the first step in the sequence are not all visible in the original configuration, and would be difficult to find. The star surgery bypasses the need to find these spheres by performing a single symplectic cut and paste operation that performs the entire sequence of rational blow-downs simultaneously.

However, there are other star surgeries which are inequivalent to any sequence of symplectic rational blow-downs as proven by a family of examples in [40]. It is expected that many of these star surgery operations cannot be obtained from sequences of rational blow-downs. This contrasts with the operations one would obtain by replacing linear plumbings of spheres by alternate fillings, which were shown by Bhupal and Özbağci [6] to all be equivalent to sequences of rational blow-downs.

Using star surgery, we construct many examples of exotic 4-manifolds. These constructions involve two steps. First we must find a configuration of symplectic spheres inside a well understood 4-manifold. In our examples we do this by looking at blow-ups of elliptic fibrations $E(1)$ using varying types of singular fibers to find symplectic spheres with the required intersection data. We explicitly construct many elliptic fibrations by blowing up various Lefschetz pencils on $\mathbb{C P}^{2}$. Then we apply the star surgery operation which replaces this neighborhood of spheres with the smallest symplectic filling of the induced contact boundary Seifert fibered space. By keeping track of the homology classes of all of the spheres in the elliptic fibration, we are able to compute the small perturbation Seiberg-Witten invariants of the resulting manifold.

Using this technique, we construct a minimal symplectic 4-manifold which is an exotic copy of $\mathbb{C P}^{2} \# 8 \overline{\mathbb{C P}^{2}}$.

Theorem 1.1 There is a minimal symplectic 4-manifold $X$ which is homeomorphic but not diffeomorphic to $\mathbb{C P}^{2} \# 8 \overline{\mathbb{C P}^{2}}$ and which is obtained by a star surgery. The symplectic Kodaira dimension of $X$ is 2 . 
We push our techniques further with different examples of star surgery operations. These examples yield exotic (potentially nonminimal) symplectic copies of $\mathbb{C P}^{2} \# k \overline{\mathbb{C P}^{2}}$, for $k=6,7$.

Theorem 1.2 There are constructions of symplectic exotic copies of $\mathbb{C P} \# 7 \overline{\mathbb{C P}^{2}}$ and $\mathbb{C P}^{2} \# 6 \overline{\mathbb{C P}^{2}}$ obtained by performing star surgery operations on blow-ups of $E(1)$.

Other star surgery operations, including the star surgery which is known to be inequivalent to any sequence of rational blow-downs, can be used for similar constructions. In particular, we show that this star surgery can be used to construct an exotic $\mathbb{C} \mathrm{P}^{2} \# 8 \overline{\mathbb{C P}^{2}}$, and related star surgeries can be used to improve these constructions to manifolds with $b_{2}^{-}=6,7$.

While the star surgery operations are inspired by symplectic topology, they can also be used smoothly in the absence of a symplectic structure. By first performing Fintushel and Stern's knot surgery in a double node neighborhood [16] (which destroys the symplectic structure), and then performing the star surgery as in Theorem 1.1, we prove the following result.

Theorem 1.3 For every $n \geq 2$ there exist smooth minimal mutually nondiffeomorphic 4-manifolds $Y_{n}$ which are all homeomorphic to $\mathbb{C P}^{2} \# 7 \overline{\mathbb{C P}^{2}}$. These manifolds are obtained by a star surgery.

The same technique of first performing knot surgery in a double node neighborhood can be used with the star surgery constructions in the proof of Theorem 1.2 to produce infinitely many smooth but not symplectic nondiffeomorphic manifolds in the homeomorphism classes of $\mathbb{C P}^{2} \# 6 \overline{\mathbb{C P}^{2}}$ and $\mathbb{C P}^{2} \# 5 \overline{\mathbb{C P}^{2}}$.

Examples of (minimal) exotic copies of $\mathbb{C P}^{2} \# k \overline{\mathbb{C P}^{2}}$, for $k=6,7,8$ have previously been constructed using the rational blow-down technique. The exotic structure was detected by calculating the effect of the rational blow-down on the Seiberg-Witten invariants; see Park [36], Stipsicz and Szabó [42], Fintushel and Stern [16], and Michalogiorgaki [30]. The effect of star surgery on Seiberg-Witten invariants is similar to the effect of rational blow-down [30]. The main reason is that the boundary of the star shaped configuration is an $L$-space. In other words the monopole Floer homology of the boundary of the neighborhood of these configurations of spheres is the simplest group it could be.

Finding exotic copies of $\mathbb{C} \mathrm{P}^{2} \# k \overline{\mathbb{C} P^{2}}$ for small $k$ is a problem which has been studied for many years. In the 1980s, gauge theoretic techniques were used to distinguish Dolgachev surfaces from $\mathbb{C P}^{2} \# 9 \overline{\mathbb{C P}^{2}}$, as in Donaldson [9] and Friedman and Morgan [20], and the Barlow surface from $\mathbb{C P}^{2} \# 8 \overline{\mathbb{C P}^{2}}$; see Kotschick [25]. Significant 
progress was made using the rational blow-down to construct an exotic $\mathbb{C P}^{2} \# k \overline{\mathbb{C P}^{2}}$ for $k \geq 5$; see Park [36], Stipsicz and Szabó [42], Park, Stipsicz and Szabó [37], Fintushel and Stern [16], and Michalogiorgaki [30]. Later this was improved to $k \geq 2$ using different techniques; see Akhmedov and Park [4; 3], Akhmedov [1], Akhmedov, Baykur and Park [2], Baldridge and Kirk [5], Fintushel and Stern [18] and Fintushel, Park and Stern [13]. Because these star surgery operations greatly increase the possible configurations of surfaces which can be cut out and replaced, we hope that more star surgery constructions will be found and can be used to improve this bound or exhibit other new phenomena in smooth 4-manifold topology.

The organization is as follows: in Section 2 we define our star surgery operation, and describe the explicit examples which we will use in constructions of exotic 4-manifolds. In Section 3, we determine properties of these star surgery operations by computing algebraic topological invariants of the fillings. In Section 4, we construct three explicit elliptic fibrations which we will use to embed configurations of symplectic spheres on which to perform star surgery. Theorems 1.1 and 1.2 are proven in Section 5, where we use star surgery to construct manifolds and compute their homeomorphism invariants, Kodaira dimension, and Seiberg-Witten invariants. Finally, Theorem 1.3 is proven in Section 6 , by using knot surgery and star surgery together.

Acknowledgments We would like to thank Kouichi Yasui and Tian-Jun Li for helpful correspondences. In the course of this work, the first author was supported by the National Science Foundation FRG Grant DMS-1065178 and a TUBITAK grant BIDEB 2232. The second author was supported by a National Science Foundation Graduate Research Fellowship under Grant No. DGE-1110007.

\section{Star surgery}

\section{A Description}

Rational blow-downs of plumbings of spheres were shown to be symplectic operations by Symington $[44 ; 45]$ proving that both the plumbing of spheres and the rational homology ball support symplectic structures with convex boundary inducing the same contact structures. One may ask more generally what can replace a neighborhood of spheres in this symplectic cut and paste manner. This question is reduced to understanding symplectic fillings of certain contact structures by the following result of Gay and Stipsicz. 
Theorem 2.1 [23, Theorem 1.2] If $C=C_{1} \cup \cdots \cup C_{n} \subset(X, \omega)$ is a collection of symplectic surfaces in a symplectic 4-manifold $(X, \omega)$ intersecting each other $\omega-$ orthogonally according to the negative-definite plumbing graph $\Gamma$ and $\nu C \subset X$ is an open set containing $C$, then $C$ admits an $\omega$-convex neighborhood $U_{C} \subset \nu C \subset(X, \omega)$.

Note that the $\omega$-orthogonal condition can be achieved by any configuration of spheres which intersect positively and transversely by an isotopy through symplectic spheres.

The contact structures induced on the boundaries can be understood through an open book decomposition by results of Gay and Mark (under the additional assumption that the plumbing graph contains no bad vertices, ie $w_{j}+e_{j}<0$, where $w_{j}$ is the weight of a vertex $v_{j}$, and $e_{j}$ is the number of edges emanating from $v_{j}$ ). Let $C$ be the union of symplectic surfaces intersecting $\omega$-orthogonally according to such a graph. Form a surface $\Sigma$ from the plumbing graph as follows. Start with the surfaces corresponding to each vertex $v_{j}$ and take the connected sum with $\left|w_{j}+e_{j}\right|$ disks. Then take the connected sum of the resulting surfaces according to the edges of the graph. Take one simple closed curve around each connected sum neck, and denote these curves by $c_{1}, \ldots, c_{k}$.

Theorem 2.2 [21, Theorem 1.1] Any neighborhood of $C$ contains a neighborhood of $C$ with strongly convex boundary that admits a symplectic Lefschetz fibration having regular fibers $\Sigma$ and exactly one singular fiber. The vanishing cycles are $c_{1}, \ldots, c_{k}$ and $C$ is the union of the closed components of the singular fiber. The induced contact structure on the boundary is supported by the induced open book $(\Sigma, \tau)$, where $\tau$ is a composition of positive Dehn twists around the curves $c_{1}, \ldots, c_{k}$.

Note that since the curves $c_{1}, \ldots, c_{k}$ are disjoint from each other, the order of the Dehn twists does not matter in defining $\tau$.

Remark 2.3 It was shown by Park and Stipsicz [34] that this contact structure is in fact the canonical contact structure on the boundary Seifert fibered space (given by the complex tangencies on the link of the corresponding normal surface singularity). Their result holds more generally for the boundary contact structure of any convex negative-definite plumbing of surfaces.

In the case that the symplectic spheres intersect according to a star-shaped graph, additional tools are available to search for alternate convex fillings of the same convex boundary. Classifications of such fillings were studied in [41]. While most plumbings do not share the same convex boundary with a rational homology ball, many share convex boundary with a symplectic filling of significantly smaller Euler characteristic. 
We will call star surgery the operation of cutting out the neighborhood of spheres which intersect according to a star-shaped graph, and replacing it with an alternative convex symplectic filling of strictly smaller Euler characteristic.

It is not clear that any contactomorphism of the boundary extends over the alternate convex filling, so the star surgery will depend on an identification of the convex contact boundary of the filling with the concave contact boundary of the complement of the star-shaped plumbing. In our cases, this identification will be made using the open book decomposition defined by Theorem 2.2 and an equivalent open book decomposition on the boundary of the alternate convex filling.

In each case, the neighborhood of spheres will be replaced by an alternate symplectic filling supported by a Lefschetz fibration. The fibers of this Lefschetz fibration will agree with the fibers of the Lefschetz fibration constructed by Gay and Mark on the plumbing neighborhood of spheres. However, the vanishing cycles will differ. We will show that the induced contact structures on the boundary agree by showing that the open book decompositions have equal monodromy. In order to do this, we will require knowledge of relations in the mapping class group of planar surfaces.

\section{B Conventions on mapping class elements and handle diagrams for Lefschetz fibrations}

A Lefschetz fibration naturally induces an open book decomposition on the boundary where the fibers of the open book are the same as the fibers of the Lefschetz fibration, and the monodromy is given by a product of positive (right-handed) Dehn twists about the vanishing cycles. Since mapping class groups of surfaces are nonabelian, the order of the vanishing cycles generally matters. Conventions in the literature vary, but we will use a fixed set of conventions throughout this paper which are consistent with each other, which we describe here.

Suppose $c_{1}, \ldots, c_{n}$ are simple closed curves on the fiber. Denote by $D_{c_{i}}$ a positive Dehn twist around $c_{i}$. The product $D_{c_{1}} D_{c_{2}} \cdots D_{c_{n}}$ means first Dehn twist along $c_{1}$, then $c_{2}$, and so on until finally along $c_{n}$, meaning we are using group notation as opposed to functional notation. When the fiber is a disk with holes, we can place the holes along a circle concentric with the boundary of the disk. Labeling the holes $\{1, \ldots, m\}$ counterclockwise, we use the notation $D_{i_{1}, \ldots, i_{k}}$ for $i_{1}, \ldots, i_{k} \in\{1, \ldots, m\}$ to indicate a positive Dehn twist about a curve which convexly contains the holes $i_{1}, \ldots, i_{k}$.

Any factorization of the monodromy of an open book decomposition into a product of positive Dehn twists corresponds to a Lefschetz fibration. When the fibers are 

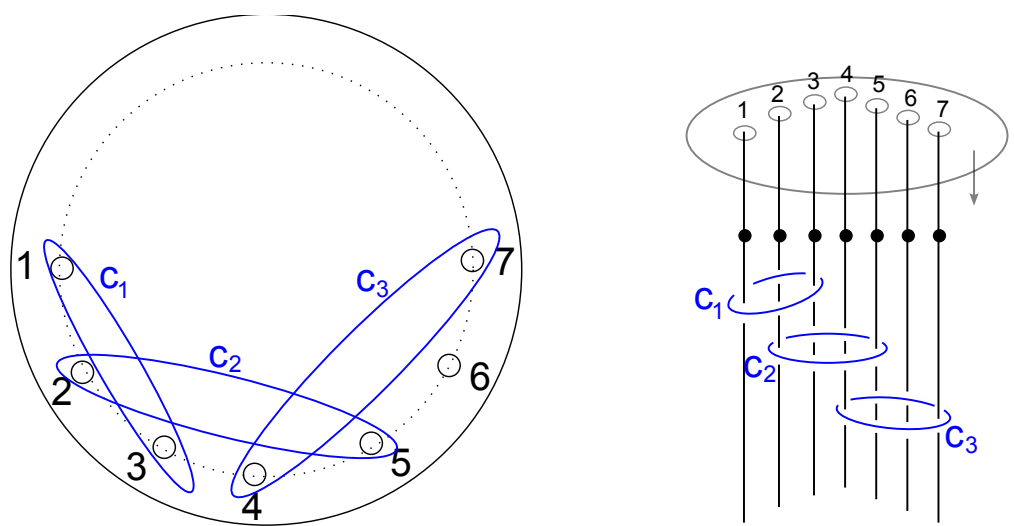

Figure 1: The Lefschetz fibration corresponding to the monodromy factorization $D_{1,3} D_{2,5} D_{4,7}=D_{c_{1}} D_{c_{2}} D_{c_{3}}$. The tops and bottoms of the dotted segments are identified to form a closed trivial braid.

disks with holes, we have the natural handlebody decomposition for this Lefschetz fibration where the holes are represented by dotted circles forming a trivial braid corresponding to 1-handles and the vanishing cycles correspond to 2-handles. We view the holed disk fibers as orthogonal to the dotted circles, oriented so that the outward normal points downward (ie turn the holed disk upside-down). Then the monodromy factorization $D_{c_{1}} \cdots D_{c_{n}}$ corresponds to the Lefschetz fibration where the vanishing cycles appear as curves, each lying in a disk transverse to the trivial braid of dotted circles such that $c_{1}$ is at the top of the diagram and $c_{n}$ at the bottom.

To draw the handlebody, we will isotope the holes on the disk so that they all lie on the bottom half of the disk along a circle concentric to the boundary. Then using the upside-down disk convention, the holes, ordered counterclockwise on the downward pointing disk, correspond to dotted trivial braid components labeled left to right. A curve which convexly encloses holes $i_{1}, \ldots, i_{k}$ will appear in this diagram as a circle, half of which passes in front of all of the dotted circles, and the other half passes behind the dotted circles corresponding to $i_{1}, \ldots, i_{k}$, but in front of all the other circles. An example, using the top to bottom convention where the outward normal to the disk points downward, is in Figure 1.

The mapping class group on a disk with holes is generated by Dehn twists. Dehn twists about disjoint curves commute. If we place the holes on a circle concentric with the boundary, we can order them counter-clockwise. Suppose $A, B$, and $C$ are collections of holes such that all the holes of $A$ precede all the holes of $B$ which precede all the holes of $C$ going around the circle counterclockwise. Then the lantern relation states

$$
D_{A \cup B \cup C} D_{A} D_{B} D_{C}=D_{A \cup B} D_{A \cup C} D_{B \cup C} .
$$


The Dehn twists on the right-hand side can be cyclically permuted.

By combining a sequence of lantern relations one obtains the daisy relation, which is given as follows. Suppose $B_{0}, B_{1}, \ldots, B_{m}$ are disjoint subsets of the $k$ holes on the disk labeled counter-clockwise $(m \geq 2)$. Then

$$
D_{B_{0} \cup B_{1} \cup \cdots \cup B_{m}} D_{B_{0}}^{m-1} D_{B_{1}} \cdots D_{B_{m}}=D_{B_{0} \cup B_{1}} D_{B_{0} \cup B_{2}} \cdots D_{B_{0} \cup B_{m}} D_{B_{1} \cup \cdots \cup B_{m}} .
$$

The daisy relations were shown to correspond to Fintushel and Stern's rational blowdown operations in [11].

We will use one more combination of lantern moves, corresponding to one of Park's generalized rational blow-downs which starts with a linear plumbing with weights $(-2,-5,-3)$. It was first worked out in [11] that the relation is given by

$$
\begin{aligned}
& D_{A \cup B \cup C \cup D \cup E} D_{A \cup B} D_{A} D_{B} D_{C}^{2} D_{D} D_{E} \\
& \quad=D_{A \cup C} D_{B \cup C} D_{A \cup B \cup D} D_{A \cup B \cup E} D_{C \cup D \cup E}
\end{aligned}
$$

for a disk whose holes are grouped into sets $A, B, C, D, E$, labeled counter-clockwise. This equality can be shown by performing one daisy relation (2-2) where $B_{0}=A \cup B$ introducing some negative Dehn twists, followed by a lantern relation (2-1).

\section{C The family of star surgeries $\left(\mathcal{S}_{i}, \mathcal{T}_{i}\right)$}

A particularly nice family of star-shaped surgeries is given by symplectically replacing a neighborhood of a configurations of spheres, $\mathcal{S}_{i}$ by its smallest filling $\mathcal{T}_{i}$ (a specific filling of minimal Euler characteristic). The configurations $\mathcal{S}_{i}$ are made up of symplectic spheres which intersect according to star-shaped graphs with $i+2$ arms. Each arm contains $i-1$ spheres of square -2 , and the central vertex is a sphere of square $-i-3$; see Figure 2(a). Note that $\mathcal{S}_{1}$ is just a -4 -sphere, and the replacement $\mathcal{T}_{1}$ is the rational blow-down of this -4 -sphere. However for $i>1$, the graphs are star-shaped but not linear, and the replacement fillings $\mathcal{T}_{i}$ are not obtained by a rational blow-down of a subgraph of the spheres shown in the original configuration. Handlebody diagrams for the fillings $\mathcal{T}_{i}$ for $i=1,2,3$ are shown in Figure 3. In general, a handlebody diagram for $\mathcal{T}_{i}$ (see Figure 9(a)) has $i+21$-handles represented by dotted circles, and $\frac{1}{2}(i+1)(i+2) 2$-handles, one passing through each distinct pair of 1 -handles. The corresponding monodromy factorization is

$$
\left(D_{1,2} D_{1,3} D_{1,4} \cdots D_{1, i+2}\right)\left(D_{2,3} D_{2,4} \cdots D_{2, i+2}\right) \cdots\left(D_{i, i+1} D_{i, i+2}\right)\left(D_{i+1, i+2}\right) .
$$

We can replace $\mathcal{S}_{i}$ by $\mathcal{T}_{i}$ symplectically due to the following proposition. 


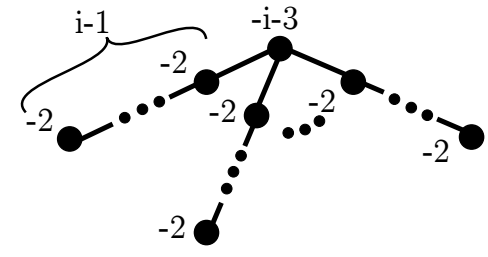

(a)

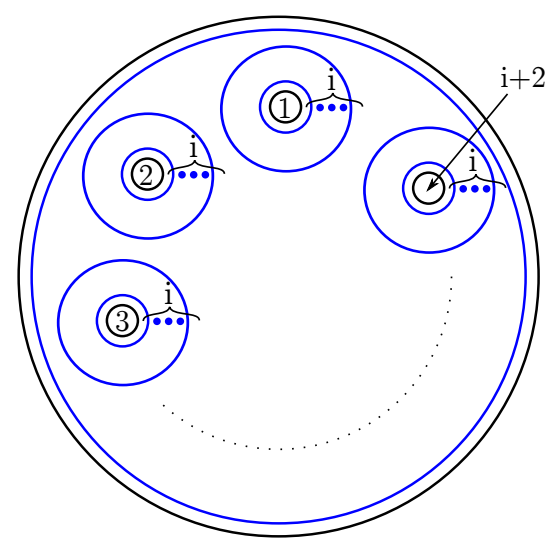

(b)

Figure 2: (a) Plumbing graph for $\mathcal{S}_{i}$ and (b) fibers and vanishing cycles for $\mathcal{S}_{i}$.

Proposition 2.4 The contact structure induced on the convex boundary of the plumbing of spheres $\mathcal{S}_{i}$ is the same as the contact structure induced on the convex boundary of $\mathcal{T}_{i}$ (whose symplectic structure is determined by the supporting Lefschetz fibration described above).

Proof For the plumbing of spheres $\mathcal{S}_{i}$, Gay and Mark's construction implies that the fibers of the supporting Lefschetz fibration are $(i+2)$-holed disks, and the vanishing cycles consist of a single curve parallel to the outer boundary component, and $i$ boundary parallel curves around each of the holes; see Figure 2(b).

In order to show that the contact structures induced on the boundaries of $\mathcal{S}_{i}$ and $\mathcal{T}_{i}$ agree, we will show that the open book decompositions induced on the boundary of the corresponding Lefschetz fibrations are the same. Since the pages are the same, it suffices to show the monodromies are equal, which amounts to the following relation:

$$
\begin{aligned}
D_{1,2, \ldots, i+1, i+2} D_{1}^{i} & \cdots D_{i+2}^{i} \\
= & \left(D_{1,2} D_{1,3} D_{1,4} \cdots D_{1, i+2}\right)\left(D_{2,3} D_{2,4} \cdots D_{2, i+2}\right) \cdots \\
& \cdots\left(D_{i, i+1} D_{i, i+2}\right)\left(D_{i+1, i+2}\right) .
\end{aligned}
$$

This relation is sometimes referred to in the literature as the generalized lantern relation. To see these are equal in the mapping class group, proceed by induction on $i$. When $i=1$ this is the standard lantern relation. By a relabeled version of the $i-1$ case, we can use the inductive hypothesis to say that the right-hand side is equal to

$$
\left(D_{1,2} D_{1,3} D_{1,4} \cdots D_{1, i+2}\right) D_{2,3, \cdots, i+1, i+2} D_{2}^{i-1} D_{3}^{i-1} \cdots D_{i+1}^{i-1} D_{i+2}^{i-1} \text {. }
$$

Applying a daisy relation to this then gives the left-hand side. 

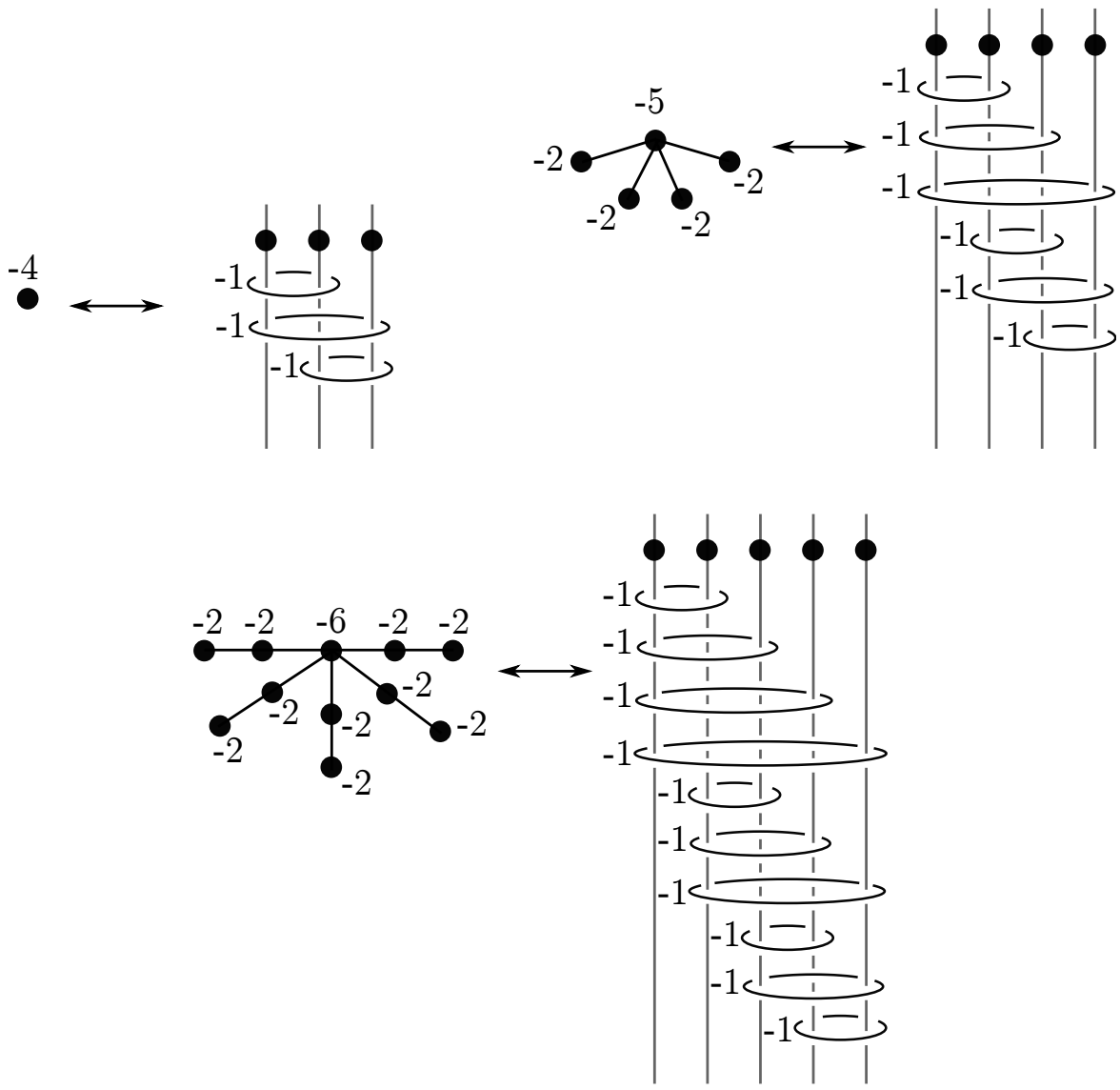

Figure 3: The first three configurations of spheres and their replacement symplectic fillings in the family $\left(\mathcal{S}_{i}, \mathcal{T}_{i}\right)$. Top left: $\mathcal{S}_{1}, \mathcal{T}_{1}$. Top right: $\mathcal{S}_{2}, \mathcal{T}_{2}$. Bottom: $\mathcal{S}_{3}, \mathcal{T}_{3}$.

Remark 2.5 For the negative-definite star plumbings we consider, the induced contact structure is supported by a planar open book. By work of Wendl [47], any other convex filling is supported by a planar Lefschetz fibration inducing the same open book decomposition on the boundary. Therefore the two Lefschetz fibrations correspond to positive factorizations of the same monodromy. Equivalent elements in a planar mapping class group are always related by some sequence of lantern relations and commutation, but this sequence may pass through factorizations involving negative Dehn twists. If one can obtain one positive factorization from another through a sequence of relations so that at each stage we remain in a positive factorization then the overall symplectic operation is broken down into a sequence of other symplectic operations. 


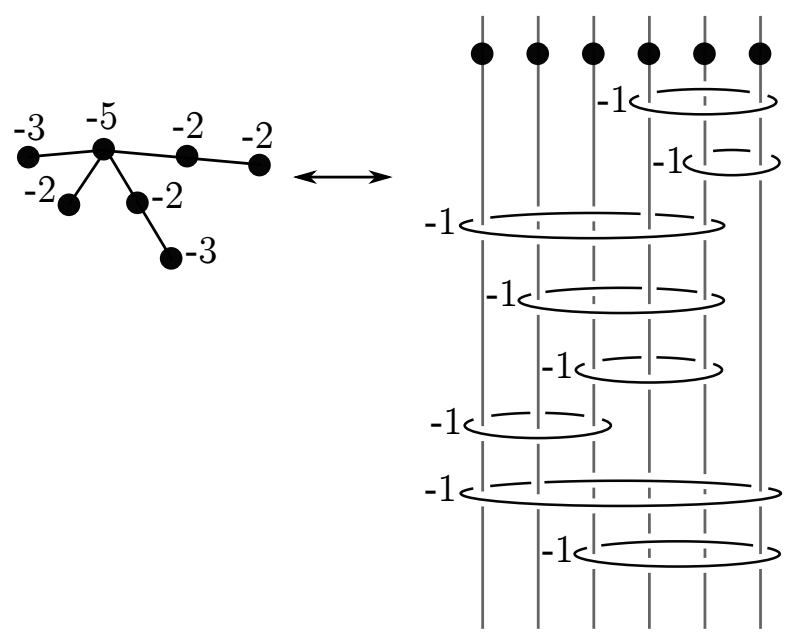

Figure 4: A star-shaped plumbing graph for $\mathcal{Q}$, and an alternate symplectic filling $\mathcal{R}$.

For example, this proof of the generalized lantern relation shows that it can be obtained by performing a sequence of daisy and lantern relations, so that after each relation, we still have a positive factorization. Endo, Mark, and Van Horn-Morris [11] showed that daisy relations correspond to Fintushel-Stern rational blow-downs. Therefore these particular star surgeries are equivalent to sequences of rational blow-downs. However, it is not easy to see the existence of all the configurations which are rationally blowndown at each stage, so in applications it would be difficult to find all of these rational blow-downs to perform. Instead, we can just perform the sequence all at once with a single star surgery.

A question one can ask is whether all such star surgery operations arise as sequences of rational blow-downs. Surprisingly, this was shown in the linear case in [6], but it was suspected that star surgery was more general. After the appearance of the first draft of this paper, the second author proved that certain examples of a star surgery cannot be realized as any sequence of symplectic rational blow-downs [40, Theorem 1.2].

\section{D The star surgery $(\mathcal{Q}, \mathcal{R})$}

The following star surgery is related to the $\mathcal{S}_{2}, \mathcal{T}_{2}$ star surgery but improves it in the sense that it reduces the Euler characteristic by a larger amount. Let $\mathcal{Q}$ denote the configuration of spheres indicated on the left-hand side of Figure 4 . Let $\xi_{\text {can }}$ be the canonical contact structure $\partial \mathcal{Q}$. 
Proposition 2.6 There exists a symplectic manifold $\mathcal{R}$ of Euler characteristic 3 with convex boundary, such that the induced contact manifold on the boundary is contactomorphic to $\left(\partial \mathcal{Q}, \xi_{\text {can }}\right)$.

Proof Let $\mathcal{R}$ be the 4-manifold given by the handlebody diagram on the right of Figure 4. This particular diagram makes apparent a Lefschetz fibration structure on $\mathcal{R}$. The fibers of the Lefschetz fibration are 6-holed disks, and the base is a disk. The fibers near the boundary are disks perpendicular to the dotted circles which give the holes. The vanishing cycles are given by the -1 -framed 2 -handles. We can verify that the induced open book decomposition on the boundary agrees with the one that is induced on the boundary of the symplectic plumbing given by [21].

We choose a standard 6-holed disk, such that the holes are centered at the vertices of a regular hexagon on the disk. Label the holes with numbers $1, \ldots, 6$ going around counter-clockwise. The construction of Gay and Mark [21] indicates that the open book induced on the boundary of the plumbing $\mathcal{Q}$ has pages which are 6-holed disks, with monodromy given by positive Dehn twists about disjoint curves enclosing holes as follows:

$$
D_{123456} D_{12} D_{1} D_{2} D_{3}^{2} D_{45}^{2} D_{4} D_{5} D_{6}^{3} \text {. }
$$

The monodromy induced by the Lefschetz fibration on $\mathcal{R}$ (reading the vanishing cycles from top to bottom) is

$$
D_{46} D_{56} D_{145} D_{245} D_{345} D_{123} D_{126} D_{36} \text {. }
$$

Commuting when needed and then performing a Park relation (2-3) on the plumbing monodromy, where $A=\{4\}, B=\{5\}, C=\{6\}, D=\{1,2\}$ and $E=\{3\}$, we get an intermediate factorization:

$$
D_{46} D_{56} D_{1245} D_{345} D_{1236} D_{45} D_{6} D_{1} D_{2} D_{3} .
$$

Note that this corresponds to a symplectic filling obtained from the original plumbing by rationally blowing down the configuration that comes from $u_{4,1}, u_{0}$ and the symplectic resolution of the union of $u_{3,1}$ with $u_{3,2}$. Continuing, by commuting terms and performing a lantern relation (2-1), where $A=\{45\}, B=\{1\}$ and $C=\{2\}$, we obtain the factorization

$$
D_{46} D_{56} D_{145} D_{245} D_{12} D_{345} D_{1236} D_{6} D_{3} \text {. }
$$

Note this corresponds to rationally blowing down a $-4-$-sphere which was not visible until after the first rational blow-down. We commute terms and perform one more lantern relation (2-1) (corresponding to blowing down another -4-sphere), where 

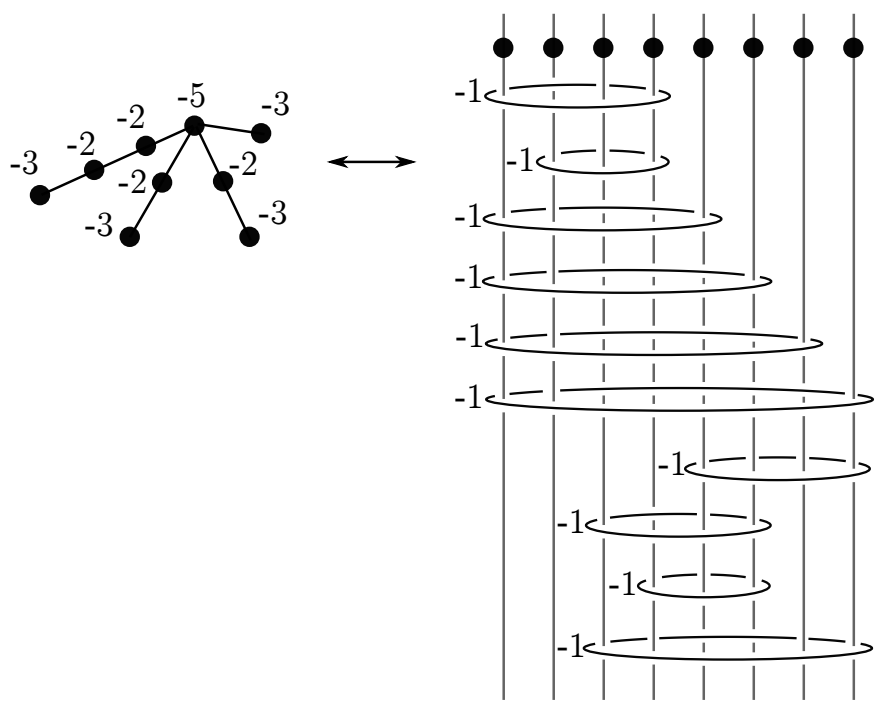

Figure 5: A star-shaped plumbing graph for $\mathcal{U}$, and an alternate symplectic filling $\mathcal{V}$.

$A=\{12\}, B=\{3\}$ and $C=\{6\}$, to obtain the factorization corresponding to the Lefschetz fibration on $T$ :

$$
D_{46} D_{56} D_{145} D_{245} D_{345} D_{123} D_{126} D_{36} \text {. }
$$

Remark 2.7 The proof makes it clear that $\mathcal{R}$ is obtained from $\mathcal{Q}$ through a sequence of rational blow-downs: one Park rational blow-down of a $(-2,-5,-3)$ configuration, followed by two consecutive rational blow-downs of -4 -spheres.

\section{E The star surgery $(\mathcal{U}, \mathcal{V})$}

Let $\mathcal{U}$ denote the plumbing according to the graph in Figure 5. Let $\xi$ denote the (canonical) contact structure induced on the convex boundary of the plumbing.

Proposition 2.8 There exists a symplectic manifold $\mathcal{V}$ of Euler characteristic 3 with convex boundary, such that the induced contact manifold on the boundary is contactomorphic to $\left(\partial \mathcal{U}, \xi_{\mathrm{can}}\right)$.

Proof Gay and Mark's construction gives an open book on the boundary of the Lefschetz fibration for the plumbing $\mathcal{U}$ whose pages are 8 -holed disks and whose monodromy is given as follows:

$$
D_{12345678} D_{12}^{3} D_{1} D_{2} D_{34}^{2} D_{3} D_{4} D_{56}^{2} D_{5} D_{6} D_{78} D_{7} D_{8} \text {. }
$$



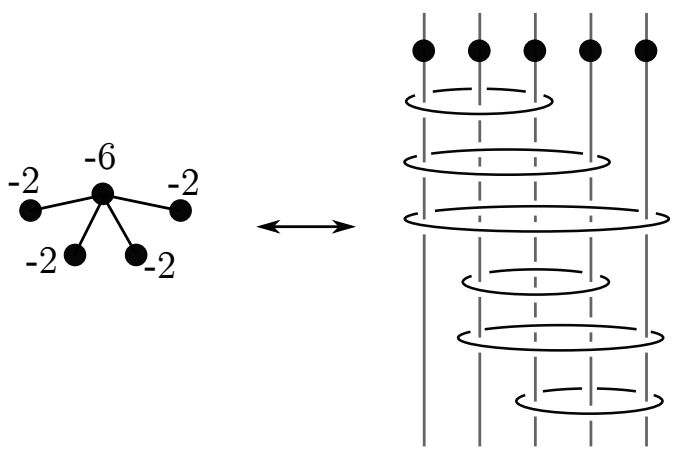

Figure 6: The $\mathcal{K}$ plumbing and alternate filling $\mathcal{L}$, providing a star surgery operation which is inequivalent to a sequence of rational blow-downs [40].

Using the monodromy equivalence corresponding to the Park $(-2,-5,-3)$ rational blow-down, where $A=\{1\}, B=\{2\}, C=\{3,4\}, D=\{5,6\}, E=\{7,8\}$, we get the following monodromy:

$$
D_{134} D_{234} D_{1256} D_{1278} D_{345678} D_{12}^{2} D_{3} D_{4} D_{56} D_{5} D_{6} D_{7} D_{8} \text {. }
$$

After commuting Dehn twists about disjoint curves, we can perform two lantern relations, one where $A=\{1,2\}, B=\{5\}$, and $C=\{6\}$ and the other where $A=\{1,2\}$, $B=\{7\}$, and $C=\{8\}$, which results in the following factorization:

$$
D_{134} D_{234} D_{125} D_{126} D_{56} D_{127} D_{128} D_{78} D_{345678} D_{3} D_{4} D_{56} \text {. }
$$

After commuting $D_{56}$ and $D_{78}$ towards the end, we can use a daisy relation with $B_{0}=\{5,6\}, B_{1}=\{7,8\}, B_{2}=\{3\}$, and $B_{3}=\{4\}$. The resulting monodromy corresponds to that of the Lefschetz fibration for $\mathcal{V}$ :

$$
D_{134} D_{234} D_{125} D_{126} D_{127} D_{128} D_{5678} D_{356} D_{456} D_{3478}
$$

Remark 2.9 Note that this proof shows that this operation is also obtained as a sequence of rational blow-downs.

\section{F The star surgeries $(\mathcal{K}, \mathcal{L}),(\mathcal{M}, \mathcal{N})$ and $(\mathcal{O}, \mathcal{P})$}

In [40], it was shown that a configuration of symplectic spheres intersecting according to a graph as in Figure 6 can be replaced by a symplectic filling of Euler characteristic two, whose Lefschetz fibration handlebody is shown in Figure 6.

The corresponding monodromy substitution for this $\mathcal{K}, \mathcal{L}$ star surgery is

$$
D_{1}^{2} D_{2}^{2} D_{3} D_{4}^{2} D_{5}^{2} D_{12345}=D_{123} D_{14} D_{15} D_{24} D_{25} D_{345}
$$




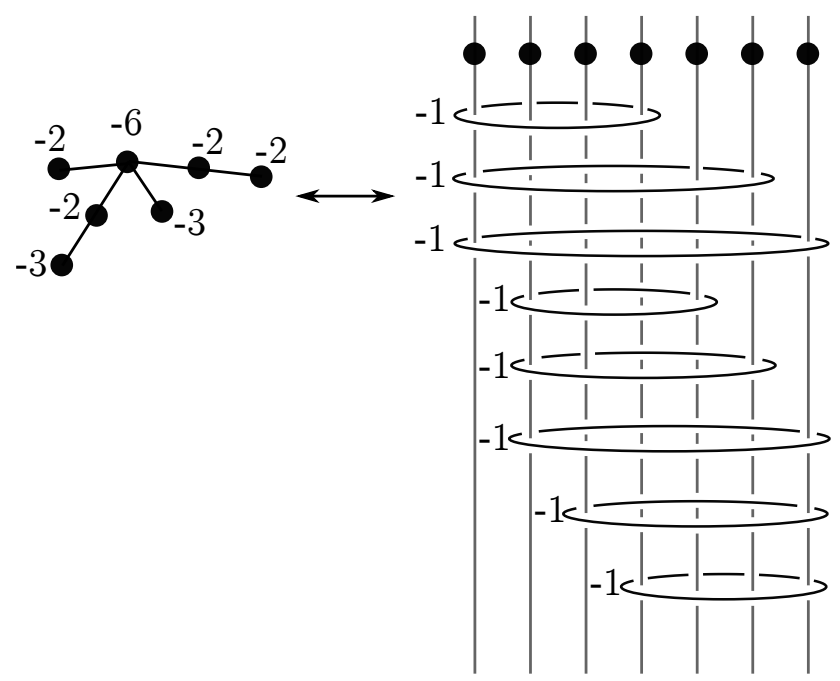

Figure 7: The $\mathcal{M}, \mathcal{N}$ star surgery. The dotted circles are labeled left to right as $1,2_{a}, 2_{b}, 3,4_{a}, 4_{b}, 5$.

The equivalence of these elements was shown directly in [40] and it was also shown that this substitution is not equivalent to any sequence of symplectic rational blowdowns/ups. From this star surgery, we can generate two other useful star surgeries, such that the alternate fillings also have Euler characteristic two. The plumbing and filling diagrams are shown in Figures 7 and 8.

These monodromy substitutions are obtained from the $\mathcal{K}, \mathcal{L}$ substitution (2-7) together with an additional lantern relation applied according to [11, Lemma 2.1], where some of the original holes split into two holes (eg hole 2 splits into holes $2_{a}$ and $2_{b}$ ). When applying the $\mathcal{K}, \mathcal{L}$ monodromy substitution, treat the split holes as a single joined hole, but when applying the lantern relations, treat them as separate holes. Performing two splittings and applying [11, Lemma 2.1] each time, we get a monodromy substitution

$$
\begin{aligned}
& D_{1}^{2} D_{2_{a} 2_{b}}^{2} D_{2_{a}} D_{2_{b}} D_{3} D_{4_{a} 4_{b}} D_{4_{a}} D_{4_{b}} D_{5}^{3} D_{12_{a} 2_{b} 34_{a} 4_{b} 5} \\
& =D_{12_{a} 2_{b}{ }_{3}} D_{14_{a} 4_{b}} D_{15} D_{2 a_{2}{ }_{b} 4_{a}} D_{2_{a} 2_{b} 4_{b}} D_{2_{a} 5} D_{2_{b} 5} D_{34_{a} 4_{b} 5}
\end{aligned}
$$

corresponding to the $\mathcal{M}, \mathcal{N}$ star surgery of Figure 7. Here, the holes are labeled 1, $2_{a}$, $2_{b}, 3,4_{a}, 4_{b}, 5$ counter-clockwise around the disk. Performing two more splits, we get the monodromy substitution

$$
\begin{array}{r}
D_{1_{a} 1_{b}}^{2} D_{1_{a}} D_{1_{b}} D_{2_{a} 2_{b}}^{2} D_{2_{a}} D_{2_{b}} D_{3} D_{4_{a} 4_{b}}^{2} D_{4_{a}} D_{4_{b}} D_{5_{a} 5_{b}}^{2} D_{5_{a}} D_{5_{b}} D_{1_{a} 1_{b} 2_{a} 2_{b} 34_{a} 4_{b} 5_{a} 5_{b}} \\
=D_{1_{a} 1_{b} 2_{a} 2_{b} 3} D_{1_{a} 4_{a} 4_{b}} D_{1_{b} 4_{a} 4_{b}} D_{1_{a} 1_{b} 5_{a}} D_{1_{a} 1_{b} 5_{b}} D_{2_{a} 2_{b} 4_{a}} D_{2_{a} 2_{b} 4_{b}} \\
D_{2_{a} 5_{a} 5_{b}} D_{2_{b} 5_{a} 5_{b}} D_{34_{a} 4_{b} 5_{a} 5_{b}}
\end{array}
$$




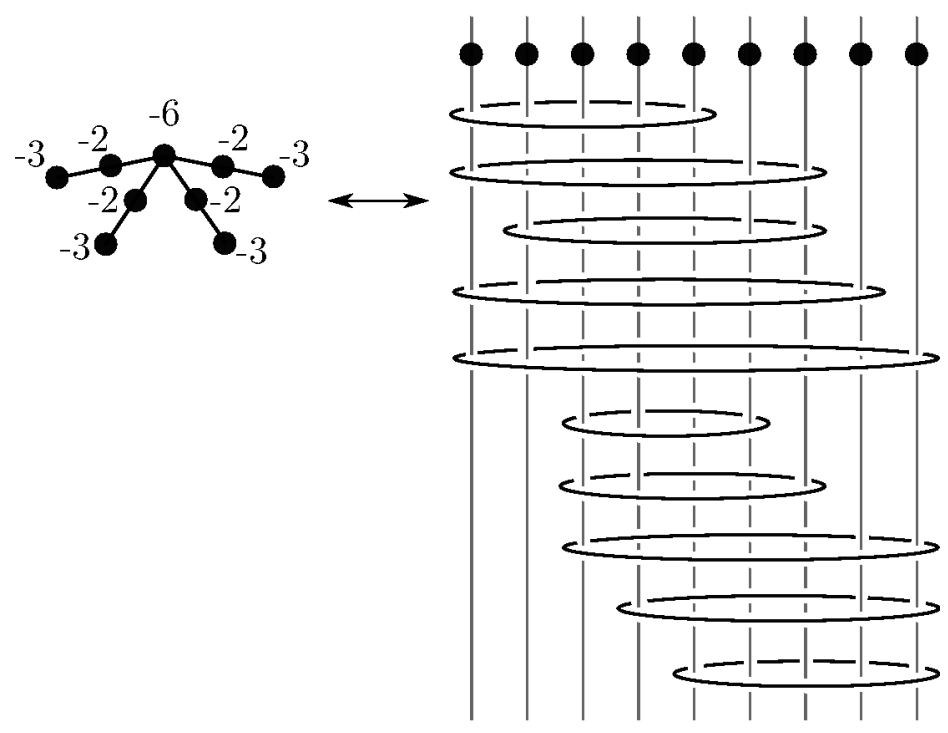

Figure 8: The $\mathcal{O}, \mathcal{P}$ star surgery. The dotted circles are labeled left to right as $1_{a}, 1_{b}, 2_{a}, 2_{b}, 3,4_{a}, 4_{b}, 5_{a}, 5_{b}$.

corresponding to the $\mathcal{O}, \mathcal{P}$ star surgery, as shown in Figure 8 on the disk with holes labeled $1_{a}, 1_{b}, 2_{a}, 2_{b}, 3,4_{a}, 4_{b}, 5_{a}, 5_{b}$ counter-clockwise. Note that the proof that these factorizations are equivalent involves applying the substitution from Equation (2-7) and then lantern relations, but the first step will introduce negative Dehn twists into the factorization, so there is not a simple way to understand these operations as a sequence of known operations (though it would be more difficult to prove that they are not equivalent to sequences of known operations; see [41; 40] for an idea of how this might be proven).

\section{Algebraic topology of the star surgery fillings}

In this section we will compute the fundamental group of the fillings $\mathcal{T}_{i}, \mathcal{R}, \mathcal{V}, \mathcal{L}$, and various other algebraic topology invariants which will be needed to understand the homeomorphism type, Kodaira dimension, and Seiberg-Witten invariants of the manifolds constructed by star surgeries using these fillings. The computations are reasonably straightforward given the handlebody descriptions of these manifolds and the Lefschetz fibration structure. The most thorough computations will be given for $\mathcal{T}_{2}$, as this will be our model example used to show how star surgery can be applied to create exotic manifolds whose Kodaira dimension and Seiberg-Witten invariants can be fully computed. 


\section{A Properties of the fillings $\mathcal{T}_{i}$}

We will compute basic algebraic topological properties of $\mathcal{T}_{i}$, and specifically $\mathcal{T}_{2}$ since we will use the $\mathcal{S}_{2}, \mathcal{T}_{2}$ star surgery to construct an exotic copy of $\mathbb{C P}^{2} \# 8 \overline{\mathrm{CP}^{2}}$.

Proposition 3.1 The fillings $\mathcal{T}_{i}$ satisfy $\pi_{1}\left(\mathcal{T}_{i}\right)=\mathbb{Z} / 2 \mathbb{Z}$. The generator can be represented by the meridian of any of the -2-framed surgery curves on the ends of the arms in $\partial \mathcal{S}_{i}$.

Proof A handlebody diagram for $\mathcal{T}_{i}$ is given as in Figure 9(a) by $(i+2)$ 1-handles corresponding to generators $\left\{y_{1}, \ldots, y_{i+2}\right\}$ of $\pi_{1}\left(\mathcal{T}_{i}\right)$, and $\frac{1}{2}(i+1)(i+2) 2$-handles corresponding to the relations $\left\{y_{j} y_{k}=1\right\}_{j \neq k \in\{1, \ldots, i+2\}}$. We can easily compute the fundamental group:

$$
\pi_{1}\left(\mathcal{T}_{i}\right)=\left\langle y_{1}, \ldots, y_{i+2}: y_{j}=y_{k}^{-1}, j \neq k\right\rangle=\left\langle y_{1}: y_{1}^{2}=1\right\rangle .
$$

Now we will track a curve representing the generator $y_{1}$ of $\pi_{1}\left(\mathcal{T}_{i}\right)$ through a sequence of equivalent surgery diagrams, to show that it restricts to a nontrivial element in $\partial \mathcal{T}_{i}=\partial \mathcal{S}_{i}$ represented by the meridian of a -2 -framed surgery curve on the end of the first arm in the standard handlebody diagram for $\mathcal{S}_{i}$. Note that $\pi_{1}$ is equivalently generated by any of the $y_{j}$ with the relation $y_{j}^{2}=1$, and that $y_{j}$ restricts in the same way to the meridian of the last curve in the $j^{\text {th }}$ arm.

Start with the handlebody diagram in Figure 9(a). The generator of the fundamental group, $y_{1}$, is represented by the dashed red curve. By rotating the plane of projection about a vertical axis, observe this handlebody diagram is isotopic to that given by Figure 9(b) (the direction into the page in Figure 9(a) corresponds to the right side of the page in Figure 9(b)). Now, exchange the dotted circles for 0 -framed circles and treat the handlebody diagram as a surgery diagram for its boundary 3-manifold. After blowing down all the -1 -framed 2-handles, we obtain a surgery diagram as in Figure 9(c) containing $i+2$ unknotted $(i+1)$-framed circles, twisted together with a full positive twist. Blowing up negatively once at a common intersection point of their Seifert surfaces, and then $i$ times along each individual curve, the curves become untwisted and 0 -framed again, so we switch the 0 -framings to dotted circles as in Figure 9(d). Perform handle slides by first sliding the dashed red curve over the top -1-framed 2-handle on that dotted circle, then sliding that 2-handle over the one below it, and so on until there is a chain of -2 curves linked to a single -1 curve. Do this for each arm (without the red reference curve on the other arms), and then finally slide the -1 -framed handle that links all of the dotted circles over each of the remaining -1-framed 2-handles. Then we see that after canceling 1,2-handle pairs, the dashed red curve appears as the meridian of the last -2 -framed curve as in Figure 9(e). 


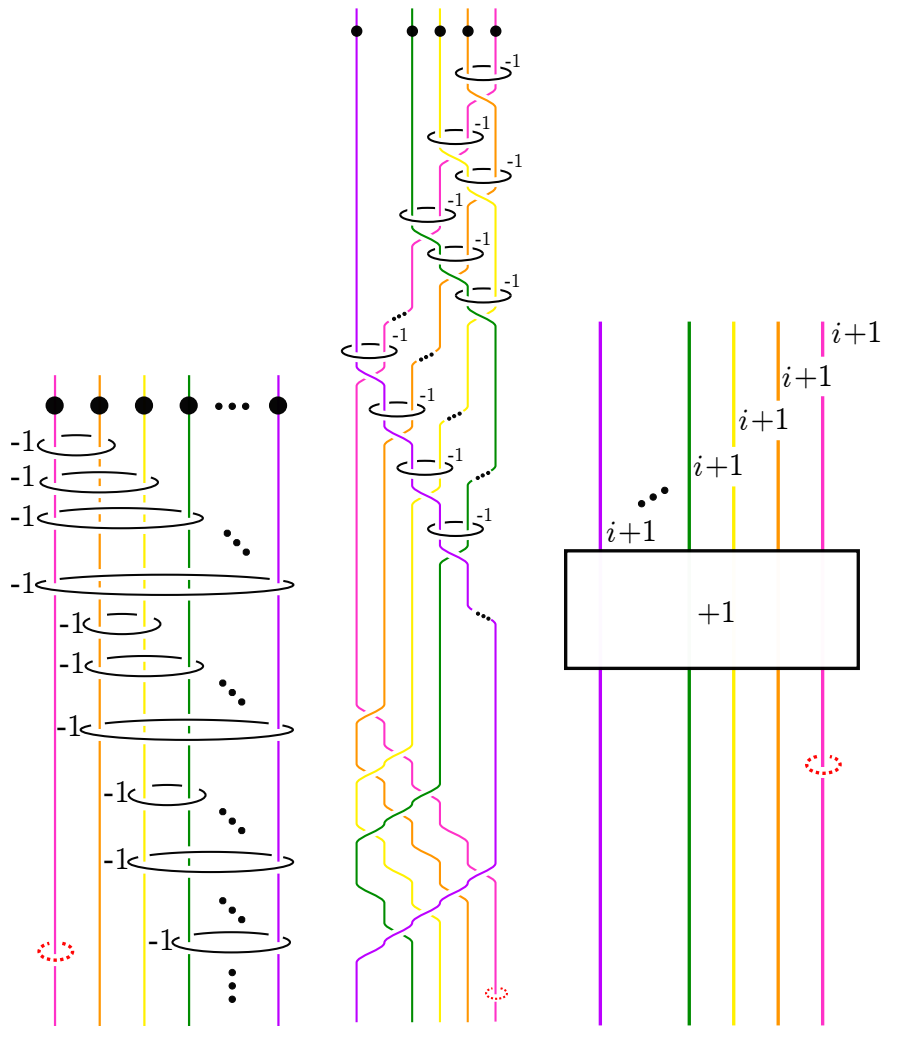

(a) (b) (c)

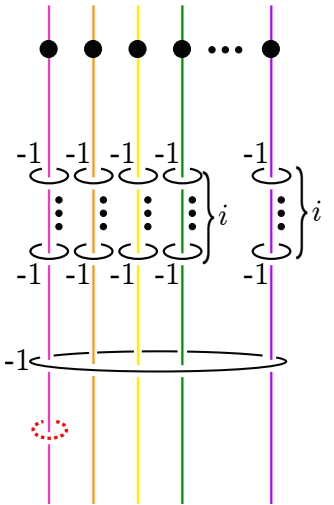

(d)

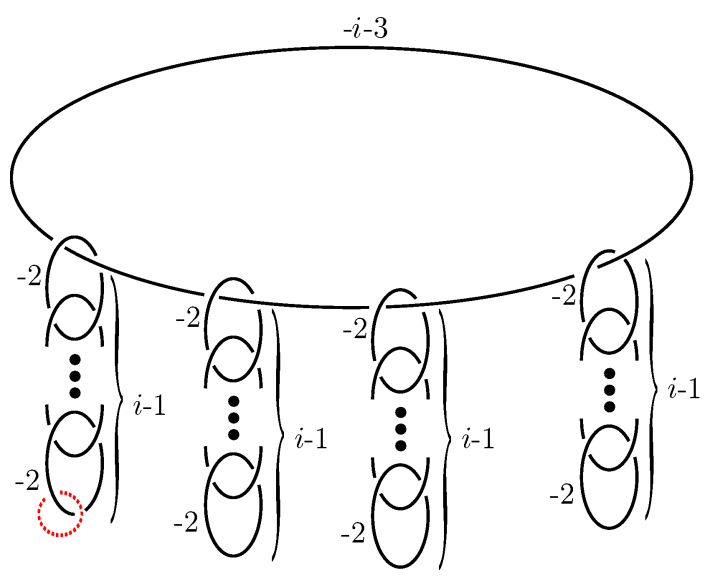

(e)

Figure 9: Relating diagrams for $\partial \mathcal{T}_{i}$ and $\partial \mathcal{S}_{i}$. We display (a) $\mathcal{T}_{i}$, (b) an isotopic diagram in a different projection, (c) the surgery diagram for the common boundary, (d) $\mathcal{S}_{i}$ Lefschetz fibration, and (e) an $\mathcal{S}_{i}$ equivalent diagram showing the plumbing. 
Proposition 3.2 The second homology of the fillings is given by $H_{2}\left(\mathcal{T}_{2} ; \mathbb{Z}\right)=\mathbb{Z} \oplus \mathbb{Z}$. The generators can be represented by tori. Moreover, $\chi\left(\mathcal{T}_{2}\right)=3$ and $\sigma\left(\mathcal{T}_{2}\right)=-2$.

Proof Using the handlebody diagram as in Figure 9(a) but ignoring the reference curve, we can compute the $\mathrm{CW}$ chain complex. Let $y_{j}$ denote the $j^{\text {th }} 1$-handle and let $x_{j k}$ denote the 2-handle whose attaching circle passes through the $j^{\text {th }}$ and $k^{\text {th }}$ 1 -handles. Then the relevant chain groups are $C_{2}\left(\mathcal{T}_{i}\right)=\left\langle x_{j k}: j \neq k \in\{1, \ldots, i+2\}\right\rangle$, $C_{1}\left(\mathcal{T}_{i}\right)=\left\langle y_{j}: j \in\{1, \ldots, i+2\}\right\rangle$. The boundary map is determined by $\partial x_{j k}=y_{j}+y_{k}$. In particular, the Euler characteristic is

$$
\chi\left(\mathcal{T}_{i}\right)=\frac{1}{2}(i+1)(i+2)-(i+2)+1 .
$$

When $i=2, \chi\left(\mathcal{T}_{2}\right)=6-4+1=3$ and the 2 -cycles are generated freely by $x_{12}+x_{34}-x_{13}-x_{24}$ and $x_{14}+x_{23}-x_{12}-x_{34}$. Note that each $x_{j k}$ has square -1 so the intersection form with respect to the above basis is given by the matrix

$$
\left[\begin{array}{rr}
-4 & 2 \\
2 & -4
\end{array}\right] \sim \mathbb{R}\left[\begin{array}{rr}
-4 & 0 \\
0 & -3
\end{array}\right]
$$

Therefore $\mathcal{T}_{2}$ is negative-definite.

We can see that the homology class $x_{12}+x_{34}-x_{13}-x_{24}$ can be represented by tori by examining the handlebody diagram. Take the cores of the 2-handles $x_{12}$ and $x_{34}$, and the cores with opposite orientation for $x_{13}$ and $x_{14}$. We connect these up to a closed torus by adding in the twice punctured disks whose outer boundary coincides with the attaching circle for $x_{i j}$ which does not intersect any of the dotted circles, and tubing together the holes with a tube encircling the dotted circle so the orientations match up as in Figure 10. One can see this surface is indeed a torus directly or check by calculating its Euler characteristic: $4 \chi\left(D^{2}\right)+4 \chi\left(2-\right.$ holed $\left.D^{2}\right)+4 \chi($ tube $)=4-4+0=0$. A similar surface represents the other generator $x_{14}+x_{23}-x_{12}-x_{34}$.

Proposition 3.3 The canonical class of the filling vanishes: $\left.K\right|_{\mathcal{T}_{2}}=0$.

Proof We will use the obstruction theoretic interpretation of the first Chern class. See [24, Proposition 2.3] and [12, Section 3.1] for related discussions. Figure 9(a) suggests that each $\mathcal{T}_{i}$ admits a positive allowable Lefschetz fibration whose fibers are disks with $i+2$ punctures, which are indicated by the circles with dots, and $\frac{1}{2}(i+2)(i+1)$ vanishing cycles which are indicated by -1 -framed $2-$ handles. This Lefschetz fibration defines an almost complex structure which is compatible with the symplectic structure of $\mathcal{T}_{i}$. We will show that $c_{1}\left(T \mathcal{T}_{i}, J\right)=0$ for $i=2$.

Let $x_{j k}$ be a $2-$ cell which is a generator of the chain complex $C_{2}\left(\mathcal{T}_{i}\right)$ as indicated in the proof of Proposition 3.2. The attaching curve $\tilde{x}_{j k}$ of each $2-$ cell $x_{j k}$ can be put on a fiber $F_{j k}$ of the Lefschetz fibration. Drawing the regular fibers on planes 


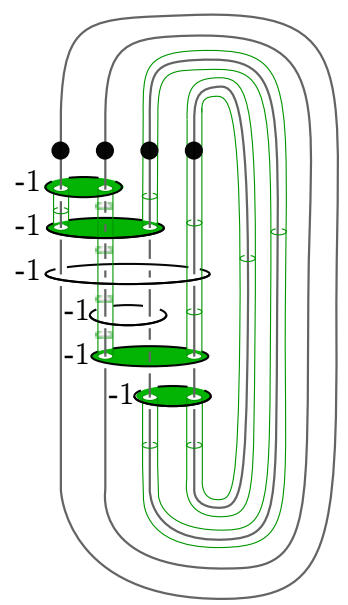

Figure 10: A torus representing a generator of the homology of $\mathcal{T}_{2}$.

induces trivializations of their tangent bundles which in turn induce a trivialization of $\left(T \mathcal{T}_{i}, J\right)$ over 1 -skeleton. Now $c_{1}\left(T \mathcal{T}_{i}, J\right)\left(x_{j k}\right)$ is the obstruction to extending this trivialization over the 2-cell $x_{j k}$. This obstruction is precisely the winding number of $\tilde{x}_{j k}$ measured with respect to the trivialization of $F_{j k}$. Each $\tilde{x}_{j k}$ is an embedded planar curve, so its winding number is one. For $i=2$ the proof of Proposition 3.2 tells us that the generators of $H_{2}\left(\mathcal{T}_{2}, \mathbb{Z}\right)$ are $x_{12}+x_{34}-x_{13}-x_{24}$ and $x_{14}+x_{23}-x_{12}-x_{34}$. Hence $c_{1}\left(T \mathcal{T}_{i}, J\right)$ evaluates as zero on both of these generators.

We will need to understand $H^{2}\left(\mathcal{T}_{2}\right)$ and $H^{2}\left(\partial \mathcal{T}_{2}\right)$, as well as the restriction map between them. By Poincaré duality, $H^{2}\left(\partial \mathcal{T}_{2}\right) \cong H_{1}\left(\partial \mathcal{T}_{2}\right)$. We can compute the first homology of the boundary of the filling explicitly from the surgery diagram obtained from the handlebody diagram of $\mathcal{T}_{2}$ by switching the dotted circles to 0 -framed circles as in Figure 11.

Proposition 3.4 Let $\lambda_{1}, \lambda_{2}, \lambda_{3}, \lambda_{4}$ denote the meridians of the four 0 -framed curves (from left to right) in the surgery diagram in Figure 11. Then

$$
\begin{aligned}
H_{1}\left(\partial \mathcal{T}_{2} ; \mathbb{Z}\right) & =\left\langle\lambda_{1}, \lambda_{2}-\lambda_{1}, \lambda_{3}-\lambda_{1} \mid 2\left(\lambda_{2}-\lambda_{1}\right)=2\left(\lambda_{3}-\lambda_{1}\right)=12 \lambda_{1}=0\right\rangle \\
& \cong \mathbb{Z} / 12 \oplus \mathbb{Z} / 2 \oplus \mathbb{Z} / 2 .
\end{aligned}
$$

Proof Let $\mu_{i j}$ denote the meridian of the -1 -framed curve which links the $i^{\text {th }}$ and $j^{\text {th }} 0$-framed curves $(i \neq j \in\{1,2,3,4\})$. 


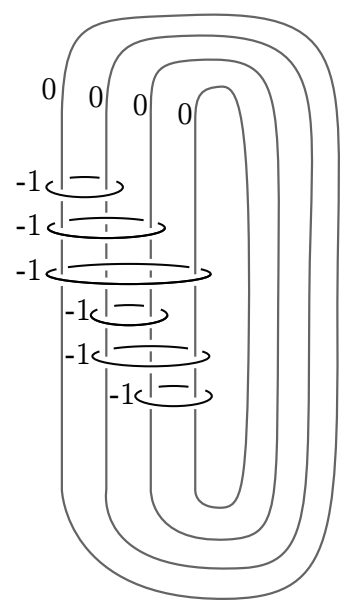

Figure 11: A surgery diagram for $\partial \mathcal{T}_{2}$.

A presentation for $H_{1}\left(\partial \mathcal{T}_{2}\right)$ has generators $\left(\lambda_{1}, \lambda_{2}, \lambda_{3}, \lambda_{4}, \mu_{12}, \mu_{13}, \mu_{14}, \mu_{23}\right.$, $\left.\mu_{24}, \mu_{34}\right)$ with relations given by the linking matrix:

$$
\begin{aligned}
-\mu_{i j}+\lambda_{i}+\lambda_{j} & =0, \\
\mu_{12}+\mu_{13}+\mu_{14} & =0, \\
\mu_{12}+\mu_{23}+\mu_{24} & =0, \\
\mu_{13}+\mu_{23}+\mu_{34} & =0, \\
\mu_{14}+\mu_{24}+\mu_{34} & =0 .
\end{aligned}
$$

Eliminate the $\mu_{i j}$ using the first relations and then solve for $\lambda_{4}$. The resulting presentation for $H_{1}\left(\partial \mathcal{T}_{2} ; \mathbb{Z}\right)$ is

$$
\left\langle\lambda_{1}, \lambda_{2}, \lambda_{3} \mid 2\left(\lambda_{2}-\lambda_{1}\right)=2\left(\lambda_{3}-\lambda_{1}\right)=-8 \lambda_{1}-2 \lambda_{2}-2 \lambda_{3}=0\right\rangle .
$$

Equivalently $\left\langle\lambda_{1}, \lambda_{2}-\lambda_{1}, \lambda_{3}-\lambda_{1} \mid 2\left(\lambda_{3}-\lambda_{1}\right)=2\left(\lambda_{2}-\lambda_{1}\right)=12 \lambda_{1}=0\right\rangle$.

\section{Proposition 3.5}

$$
H^{2}\left(\mathcal{T}_{2}\right) \cong \mathbb{Z} \oplus \mathbb{Z} \oplus \mathbb{Z} / 2
$$

Proof The $d$-cells of $\mathcal{T}^{2}$ are generators for the $d^{\text {th }} \mathrm{CW}$ homology chain complex. Their duals freely generate $C^{d}\left(\mathcal{T}_{2}\right)$. Let $y_{1}, y_{2}, y_{3}, y_{4}$ denote the 1 -handles in the diagram for $\mathcal{T}_{2}$ (from left to right), and let $x_{i j}$ denote the 2-handle whose attaching circle passes over $y_{i}$ and $y_{j}$. Let $\phi^{i}$ denote the dual of $y_{i}$, and $\psi^{i j}$ denote the dual of $x_{i j}$ so $C^{1}\left(\mathcal{T}_{2}\right)=\left\langle\phi^{1}, \phi^{2}, \phi^{3}, \phi^{4}\right\rangle$ and $C^{2}\left(\mathcal{T}_{2}\right)=\left\langle\psi^{12}, \psi^{13}, \psi^{14}, \psi^{23}, \psi^{24}, \psi^{34}\right\rangle$ and $C^{k}\left(\mathcal{T}^{2}\right)=0$ for $k>2$. Then

$$
\left(\delta \phi^{i}\right)\left(x_{j k}\right)=\phi^{i}\left(\partial x_{j k}\right)=\phi^{i}\left(y_{j}+y_{k}\right)=\delta_{j}^{i}+\delta_{k}^{i}=\left(\sum_{\ell \neq i} \psi^{i \ell}\right)\left(x_{j k}\right) \text {. }
$$


Therefore $\delta \phi^{i}=\sum_{\ell \neq i} \psi^{i \ell}$. (Note we identify $\psi^{i j}=\psi^{j i}$.)

We conclude

$$
\begin{array}{r}
H^{2}\left(\mathcal{T}_{2}\right)=\left\langle\psi^{12}, \psi^{13}, \psi^{14}, \psi^{23}, \psi^{24}, \psi^{34}\right| \psi^{12}+\psi^{13}+\psi^{14}=\psi^{12}+\psi^{23}+\psi^{24} \\
\left.=\psi^{13}+\psi^{23}+\psi^{34}=\psi^{14}+\psi^{24}+\psi^{34}=0\right\rangle .
\end{array}
$$

By eliminating variables we obtain

$$
H^{2}\left(\mathcal{T}_{2}\right)=\left\langle\psi^{12}, \psi^{13}, \psi^{12}+\psi^{13}+\psi^{23} \mid 2\left(\psi^{12}+\psi^{13}+\psi^{23}\right)=0\right\rangle \cong \mathbb{Z} \oplus \mathbb{Z} \oplus \mathbb{Z} / 2 .
$$

Proposition 3.6 The image of the restriction map $i: H^{2}\left(\mathcal{T}_{2}\right) \rightarrow H^{2}\left(\partial T_{2}\right)$ has index 2 (therefore order 24).

Proof The restriction map $i: H^{2}\left(\mathcal{T}_{2}\right) \rightarrow H^{2}\left(\partial \mathcal{T}_{2}\right)$ composed with Poincaré duality yields a map $\rho=\mathrm{PD} \circ i: H^{2}\left(\mathcal{T}_{2}\right) \rightarrow H_{1}\left(\partial \mathcal{T}_{2}\right)$. On generators $\psi^{i j}$ we have $\rho\left(\psi^{i j}\right)=$ $\mu_{i j}$, where $\mu_{i j}$ is the meridian of the surgery curve corresponding to the attaching circle for $x_{i j}$. In $H_{1}\left(\partial \mathcal{T}_{2}\right)$ we had the relation $\mu_{i j}=\lambda_{i}+\lambda_{j}$ so

$$
\begin{gathered}
\rho\left(\psi^{12}\right)=\left(\lambda_{2}-\lambda_{1}\right)+2 \lambda_{1}, \quad \rho\left(\psi^{13}\right)=\left(\lambda_{3}-\lambda_{1}\right)+2 \lambda_{1}, \\
\rho\left(\psi^{12}+\psi^{13}+\psi^{23}\right)=2\left(\lambda_{2}-\lambda_{1}\right)+2\left(\lambda_{3}-\lambda_{1}\right)+6 \lambda_{1}=6 \lambda_{1} \in H_{1}\left(\partial \mathcal{T}_{2}\right) .
\end{gathered}
$$

Therefore the image of $\rho$ (which equals the image of $i$ ) is generated by $\left(\lambda_{2}-\lambda_{1}, \lambda_{3}-\right.$ $\left.\lambda_{1}, 2 \lambda_{1}\right)$, which has index 2 in $H_{1}\left(\partial \mathcal{T}_{2}\right)$.

\section{B Properties of $\mathcal{R}$}

Lemma 3.7 The filling $\mathcal{R}$ is simply connected.

Proof Using the handlebody decomposition, a presentation for $\pi_{1}(\mathcal{R})$ is

$$
\begin{aligned}
\left\langle y_{1}, \ldots, y_{6}\right| y_{3} y_{6}=y_{4} y_{6}=y_{5} y_{6}=y_{1} y_{4} y_{5} & =y_{2} y_{4} y_{5} \\
& \left.=y_{3} y_{4} y_{5}=y_{1} y_{2} y_{3}=y_{1} y_{2} y_{6}=1\right\rangle .
\end{aligned}
$$

We can eliminate $y_{1}, y_{2}, y_{3}, y_{4}, y_{5}$ with the first five relations, resulting in the presentation

$$
\left\langle y_{6} \mid y_{6}^{-3}=y_{6}^{3}=y_{6}^{5}=1\right\rangle .
$$

Since 3 and 5 are relatively prime, this is a presentation for the trivial group.

Lemma 3.8 The intersection form on $H_{2}(\mathcal{R} ; \mathbb{Z})$ is negative-definite. In fact, there exist homology classes $\alpha$ and $\beta$ which freely generate $H_{2}(\mathcal{R} ; \mathbb{Z}) \cong \mathbb{Z}^{2}$, such that the intersection form with respect to the basis $\langle\alpha, \beta\rangle$ for $H_{2}(\mathcal{R} ; \mathbb{Z})$ is given by

$$
\left[\begin{array}{rr}
-10 & -23 \\
-23 & -79
\end{array}\right]
$$


Proof Labeling the 2-handles from top to bottom as $x_{1}, \ldots, x_{8}$, the cycles generating homology from the $\mathrm{CW}$ complex given by the handlebody decomposition are $\alpha=$ $x_{1}+x_{2}-x_{3}-x_{4}+x_{5}+x_{6}-2 x_{8}$ and $\beta=x_{1}+x_{2}-3 x_{3}-3 x_{4}+5 x_{5}+3 x_{7}-5 x_{8}$. Since $x_{i}^{2}=-1$ and the $x_{i}$ are orthogonal with respect to the intersection pairing, the intersection form can be computed directly.

\section{C Properties of $\mathcal{V}$}

Lemma 3.9 The filling $\mathcal{V}$ is simply connected.

Proof Using the handlebody decomposition, a presentation for $\pi_{1}(\mathcal{V})$ is

$$
\begin{aligned}
\left\langle y_{1}, \ldots, y_{8}\right| y_{1} y_{3} y_{4}=y_{2} y_{3} y_{4} & =y_{1} y_{2} y_{5}=y_{1} y_{2} y_{6}=y_{1} y_{2} y_{7}=y_{1} y_{2} y_{8} \\
& \left.=y_{5} y_{6} y_{7} y_{8}=y_{3} y_{5} y_{6}=y_{4} y_{5} y_{6}=y_{3} y_{4} y_{7} y_{8}=1\right\rangle .
\end{aligned}
$$

Using the first six relations to eliminate $y_{1}, y_{2}, y_{5}, y_{6}, y_{7}, y_{8}$, this simplifies to

$$
\left\langle y_{3}, y_{4} \mid\left(y_{3} y_{4}\right)^{8}=y_{3}\left(y_{3} y_{4}\right)^{4}=y_{4}\left(y_{3} y_{4}\right)^{4}=\left(y_{3} y_{4}\right)^{5}=1\right\rangle \text {. }
$$

Since 5 and 8 are relatively prime, $y_{3} y_{4}=1$. Thus $y_{3}=y_{4}=1$ so the group is trivial.

Lemma 3.10 The intersection form on $H_{2}(\mathcal{V} ; \mathbb{Z})$ is negative-definite. Moreover, $\mathrm{H}_{2}(\mathcal{V} ; \mathbb{Z}) \cong \mathbb{Z}^{2}$ and the intersection form with respect to a basis is

$$
\left[\begin{array}{cc}
-30 & 5 \\
5 & -49
\end{array}\right]
$$

Proof Labeling the 2-handles from top to bottom as $x_{1}, \ldots, x_{10}$, two cycles generating homology from the $\mathrm{CW}$ complex given by the handlebody decomposition are $-2 x_{1}-2 x_{2}+x_{3}+x_{4}-3 x_{7}+x_{8}+x_{9}+3 x_{10}$ and $-3 x_{3}-3 x_{4}+3 x_{5}+3 x_{6}-x_{7}+$ $2 x_{8}+2 x_{9}-2 x_{10}$. Since $x_{i}^{2}=-1$ and the $x_{i}$ are orthogonal with respect to the intersection pairing, the intersection form can be computed directly.

\section{D Properties of $\mathcal{L}$}

We compute the fundamental group and second homology of the filling $\mathcal{L}$ of Figure 6 .

Proposition 3.11 $\pi_{1}(\mathcal{L}) \cong \mathbb{Z} / 4$, and the generator restricts to the boundary Seifert fibered space as a meridian of any of the -2-surgery curves in the plumbing diagram. 
Proof Using the handlebody decomposition of Figure 6, we obtain a presentation for $\pi_{1}(\mathcal{L})$ generated by the five 1 -handles with relations given by the 2 -handles as follows:

$$
\left\langle y_{1}, y_{2}, y_{3}, y_{4}, y_{5} \mid y_{1} y_{2} y_{3}=y_{1} y_{4}=y_{1} y_{5}=y_{2} y_{4}=y_{2} y_{5}=y_{3} y_{4} y_{5}=1\right\rangle,
$$

which simplifies to

$$
\left\langle y_{1}, y_{2}, y_{3} \mid y_{1} y_{2} y_{3}=y_{2} y_{1}^{-1}=y_{3} y_{2}^{-2}=1\right\rangle,
$$

which again simplifies to

$$
\left\langle y_{1} \mid y_{1}^{4}=1\right\rangle \text {. }
$$

Note that the relations set $y_{1}, y_{2}, y_{4}^{-1}$ and $y_{5}^{-1}$ all equal. Each of these curves can be isotoped into the boundary of $\mathcal{L}$. By performing blow-downs, handle-slides, and handle cancellations, in a similar manner to Proposition 3.1, we see that these curves in $\partial \mathcal{L}$ are meridians of the -2 -surgery curves in the diagram for $\partial \mathcal{K}$.

Proposition 3.12 $H_{2}(\mathcal{L}) \cong \mathbb{Z}$. It is generated by an element $x_{14}-x_{15}-x_{24}+x_{25}$ of square -4 , represented by a torus obtained by gluing tubes to the cores of the specified 2-handles $x_{i j}$ which link the $i^{\text {th }}$ and $j^{\text {th }}$ dotted circles in Figure 6.

The proof is a direct computation from the handlebody decomposition of Figure 6 .

Proposition 3.13 Using the symplectic structure induced by the Lefschetz fibration on $\mathcal{L}$, we have $c_{1}(\mathcal{L})=0$, therefore the canonical class is trivial.

Proof This follows from the winding number interpretation of $c_{1}(\mathcal{L})$ for Lefschetz fibrations, and the fact that the generator of $H_{2}(\mathcal{L})$ passes over two Lefschetz 2-handles with +1 multiplicity and two $2-$ handles with -1 multiplicity.

\section{Elliptic fibrations}

In order to find symplectic embeddings of the plumbings into well-understood symplectic 4-manifolds, we will use many different elliptic fibrations exhibiting various types of singular fibers. These fibrations were classified by Persson in [38], providing a full list of possible configurations of singular fibers. However, in order to keep track of homology classes of symplectic spheres, we need to explicitly construct these elliptic fibrations by blowing up a special Lefschetz pencil in $\mathbb{C P}^{2}$. 
Lemma 4.1 There is an elliptic fibration on $E(1)=\mathbb{C P}^{2} \# 9 \overline{\mathbb{C P}^{2}}$ with one $I_{3}$ fiber, one $I_{0}^{*}$ fiber, three fishtail fibers, and a section. Labeling the components of the $I_{0}^{*}$ fiber as $S_{1}, \ldots, S_{5}$ where $S_{5}$ intersects the $E_{9}$ section, and $S_{4}$ intersects $S_{1}, S_{2}, S_{3}$ and $S_{5}$, and labeling the components of the $I_{3}$ fiber as $V_{1}, V_{2}, V_{3}$ where $V_{1}$ intersects the $E_{9}$ section, the homology classes are as follows:

$$
\begin{array}{ll}
{\left[S_{1}\right]=h-e_{1}-e_{2}-e_{3},} & {\left[V_{1}\right]=h-e_{1}-e_{8}-e_{9},} \\
{\left[S_{2}\right]=h-e_{1}-e_{4}-e_{5},} & {\left[V_{2}\right]=h-e_{2}-e_{4}-e_{6},} \\
{\left[S_{3}\right]=h-e_{1}-e_{6}-e_{7},} & {\left[V_{3}\right]=h-e_{3}-e_{5}-e_{7},} \\
{\left[S_{4}\right]=e_{1}-e_{8},} & \\
{\left[S_{5}\right]=e_{8}-e_{9} .} &
\end{array}
$$

Proof Let $C_{1}$ be a reducible degree-three algebraic curve in $\mathbb{C} P^{2}$ defined by a homogeneous polynomial $p_{1}$ and made up of three complex projective lines $L_{1}$, $L_{2}$ and $L_{3}$, which share a single common intersection point $p \in \mathbb{C} P^{2}$. Let $C_{2}$ be a reducible degree-three algebraic curve defined by a homogeneous polynomial $p_{2}$ and made up of three lines $L_{4}, L_{5}$ and $L_{6}$, such that $L_{4}$ passes through $p$, and $L_{5}$ and $L_{6}$ each intersect all other $L_{i}$ generically in double points; see Figure 12(a). Note that the homology class of $L_{i}$ for $i=1, \ldots, 6$ is the generator of $H_{2}\left(\mathbb{C P}^{2} ; \mathbb{Z}\right), h$. Define a Lefschetz pencil on $\mathbb{C P}^{2}$ by setting $C_{\left[t_{1}: t_{2}\right]}=\left\{t_{1} p_{1}+t_{2} p_{2}=0\right\}$. Note the base locus is the set of points where $C_{1}$ intersects $C_{2}$.

Blow up at $p$, and let the exceptional sphere represent the homology class $e_{1}$. Then the proper transforms of $L_{1}, L_{2}, L_{3}$ and $L_{4}$ represent $h-e_{1}$ in homology, and $L_{5}$ and $L_{6}$ are unchanged; see Figure 12 (b). Therefore the proper transform $\widetilde{C}_{1}$ represents $3 h-3 e_{1}$ in homology and the proper transform $\widetilde{C}_{2}$ represents $3 h-e_{1}$. We redefine $C_{1}$ to be the curve given by $\widetilde{C}_{1}$ together with the exceptional class $E_{1}$ with multiplicity two, and let $C_{2}=\widetilde{C}_{2}$; see Figure $12(\mathrm{c})$. Then the curves $C_{1}$ and $C_{2}$ represent the same class in homology so they define a new Lefschetz pencil as before.

Now blow up at the six intersection points of $L_{1}, L_{2}, L_{3}$ with $L_{5}, L_{6}$, so that the exceptional classes are labeled $e_{2}, \ldots, e_{7}$; see Figure $12(\mathrm{~d})$. Redefine $C_{1}$ and $C_{2}$ as the proper transforms, and note that these curves both represent the homology class $3 h-e_{1}-e_{2}-\cdots-e_{7}$ and thus define a Lefschetz pencil.

There is still a nonempty base locus since the exceptional sphere $E_{1}$ is now part of $C_{1}$ which intersects the proper transform of $L_{4}$. We blow up at this point to obtain a new exceptional sphere $E_{8}$; see Figure 12(e). The proper transform of $E_{1}$ represents $e_{1}-e_{8}$, so the proper transform of $C_{1}$ represents $3 h-e_{1}-e_{2}-\cdots-e_{7}-2 e_{8}$ whereas the proper transform of $C_{2}$ represents $3 h-e_{1}-e_{2}-\cdots-e_{8}$. Redefining $C_{1}$ as its 


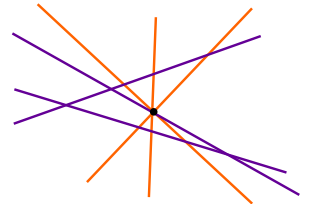

(a) Starting configuration.

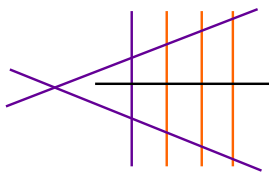

(b) Blow up $e_{1}$.

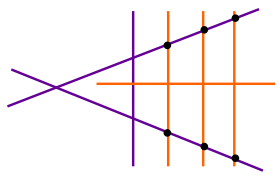

(c) Reset $C_{1}, C_{2}$.

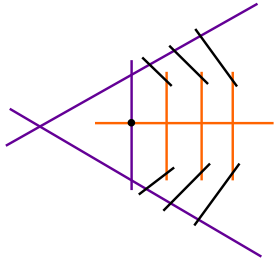

(d) Blow up $e_{2}, \ldots e_{7}$.

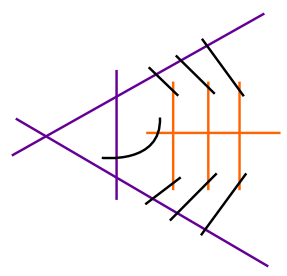

(e) Blow up $e_{8}$.

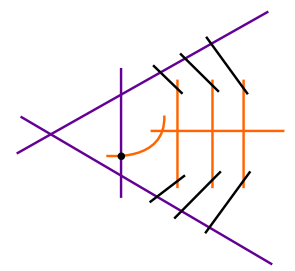

(f) Reset $C_{1}, C_{2}$.

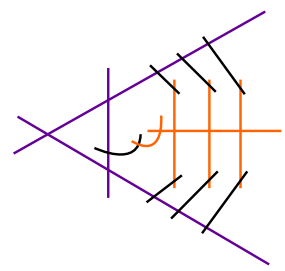

(g) Blow up $e_{9}$.

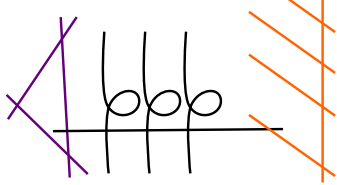

(h) Singular fibers and section.

Figure 12: Blowing up the Lefschetz pencil to a fibration. The orange curves represent $C_{1}$ and the purple curves represent $C_{2}$.

proper transform together with the exceptional sphere $E_{8}$ with multiplicity one, and $C_{2}$ as its proper transform, the two curves again define a Lefschetz pencil, but still intersect where $E_{8}$ meets $L_{4}$; see Figure $12(\mathrm{f})$. We blow up one more time at this point, and the resulting proper transforms of $C_{1}$ and $C_{2}$ are homologous and do not intersect: Figure 12(g).

The resulting elliptic fibration has singular fibers $C_{1}$ and $C_{2}$ which are of type $I_{0}^{*}$ and $I_{3}$ respectively, as well as other singular fibers, which we can perturb to generic fishtail fibers. An Euler characteristic computation implies that there are three fishtail fibers. A schematic for the singular fibers in this elliptic fibration together with the section $E_{9}$ is given by Figure 12(h).

Next, we will produce an elliptic fibration on $E(1)$ with two $I_{2}$ fibers and two $I_{4}$ fibers, and specify the homology classes of the spheres in the singular fibers.

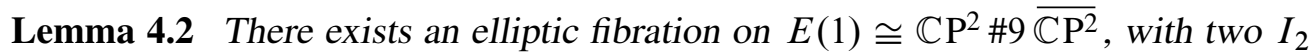
fibers and two $I_{4}$ fibers such that the spheres in these singular fibers represent homology classes as follows:

- The first $I_{2}$ fiber is made up of two -2 -spheres $\widetilde{C}_{1}$ and $\widetilde{L}_{1}$ such that

$$
\left[\widetilde{C}_{1}\right]=2 h-e_{1}-e_{2}-e_{3}-e_{4}-e_{7}-e_{8}, \quad\left[\tilde{L}_{1}\right]=h-e_{5}-e_{6}-e_{9} .
$$


- The second $I_{2}$ fiber is made up of two -2 -spheres $\widetilde{C}_{2}$ and $\widetilde{L}_{2}$ such that

$$
\left[\widetilde{C}_{2}\right]=2 h-e_{1}-e_{2}-e_{3}-e_{4}-e_{5}-e_{6}, \quad\left[\tilde{L}_{2}\right]=h-e_{7}-e_{8}-e_{9} .
$$

- The first $I_{4}$ fiber is made up of four-2-spheres, $\tilde{X}_{3}, \widetilde{Y}_{3}, \widetilde{Z}_{3}, \widetilde{E}_{3}$ with homology classes

$\left[\tilde{X}_{3}\right]=h-e_{3}-e_{6}-e_{8}, \quad\left[\tilde{Y}_{3}\right]=h-e_{3}-e_{5}-e_{7}, \quad\left[\widetilde{Z}_{3}\right]=h-e_{1}-e_{2}-e_{9}, \quad\left[\widetilde{E}_{3}\right]=e_{3}-e_{4}$.

- The second $I_{4}$ fiber is made up of four-2-spheres, $\tilde{X}_{4}, \tilde{Y}_{4}, \widetilde{Z}_{4}, \widetilde{E}_{4}$ with homology classes

$\left[\tilde{X}_{4}\right]=h-e_{1}-e_{6}-e_{7}, \quad\left[\tilde{Y}_{4}\right]=h-e_{1}-e_{5}-e_{8}, \quad\left[\tilde{Z}_{4}\right]=h-e_{3}-e_{4}-e_{9}, \quad\left[\widetilde{E}_{4}\right]=e_{1}-e_{2}$.

Moreover, this fibration admits a section whose homology class is $e_{2}$. See Figure 13 for an illustration.

Proof Consider the degree-three homogeneous polynomials in three complex variables $p_{1}(x, y, z)=\left(y z-x^{2}\right)(y-z)$ and $p_{2}(x, y, z)=\left(y z+x^{2}\right)(y+z)$. Now define $p_{\left(t_{1}, t_{2}\right)}(x, y, z)=t_{1} p_{1}(x, y, z)+t_{2} p_{2}(x, y, z)$. Then the degree-three curves $T_{\left[t_{1}: t_{2}\right]}:=\left\{p_{\left(t_{1}, t_{2}\right)}=0\right\} \subset \mathbb{C} P^{2}$ are the fibers of a Lefschetz pencil on $\mathbb{C P}^{2}$. Observe that $T_{[1: 0]}=\left\{p_{1}=0\right\}$ and $T_{[0: 1]}=\left\{p_{2}=0\right\}$ are reducible curves each made up of a linear part and an irreducible quadratic part. Let $C_{1}=\left\{y z-x^{2}=0\right\}$ denote the quadratic part of $T_{[1: 0]}$ and $L_{1}=\{y-z=0\}$ denote the linear part. Similarly, let $C_{2}=\left\{y z+x^{2}=0\right\}$ and $L_{2}=\{y+z=0\}$ denote the quadratic and linear parts of $T_{[0: 1]}$ respectively.

We will keep track of two more fibers in this Lefschetz fibration:

$$
T_{[-1: 1]}=\{(x+i z)(x-i z) y=0\}, \quad T_{[1: 1]}=\{(y+i x)(y-i x) z=0\} .
$$

These are reducible curves, each made up of three linear parts which we will label as $X_{3}=\{x+i z=0\}, Y_{3}=\{x-i z=0\}, Z_{3}=\{y=0\}, X_{4}=\{x+i y=0\}$, $Y_{4}=\{x-i y=0\}$, and $Z_{4}=\{z=0\}$.

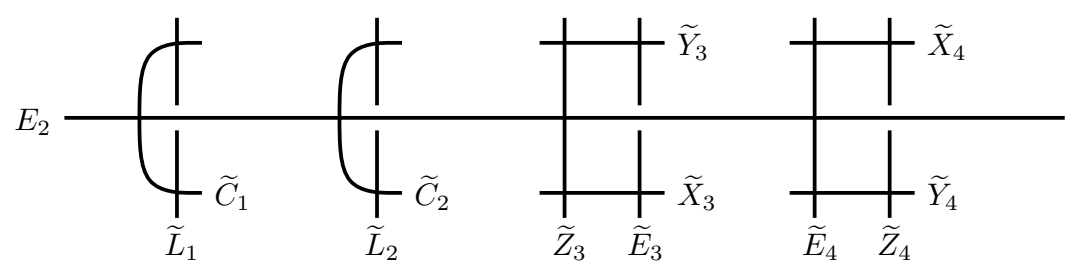

Figure 13: An elliptic fibration with two $I_{2}$ fibers and two $I_{4}$ fibers. 


\begin{tabular}{c|c|c|c|c|c|c|c|c} 
& $C_{2}$ & $L_{2}$ & $X_{3}$ & $Y_{3}$ & $Z_{3}$ & $X_{4}$ & $Y_{4}$ & $Z_{4}$ \\
\hline$C_{1}$ & {$[0: 0: 1]_{2}$} & {$[i:-1: 1]$} & {$[0: 1: 0]$} & {$[0: 1: 0]$} & {$[0: 0: 1]_{2}$} & {$[0: 0: 1]$} & {$[0: 0: 1]$} & {$[0: 1: 0]_{2}$} \\
& {$[0: 1: 0]_{2}$} & {$[-i:-1: 1]$} & {$[-i:-1: 1]$} & {$[i:-1: 1]$} & & {$[i:-1: 1]$} & {$[-i:-1: 1]$} & \\
\hline$L_{1}$ & {$[i: 1: 1]$} & {$[1: 0: 0]$} & {$[-i: 1: 1]$} & {$[i: 1: 1]$} & {$[1: 0: 0]$} & {$[-i: 1: 1]$} & {$[i: 1: 1]$} & {$[1: 0: 0]$} \\
& {$[-i: 1: 1]$} & & & & & & & \\
\hline$C_{2}$ & & & {$[0: 1: 0]$} & {$[0: 1: 0]$} & {$[0: 0: 1]_{2}$} & {$[0: 0: 1]$} & {$[0: 0: 1]$} & {$[0: 1: 0]_{2}$} \\
& & & {$[-i: 1: 1]$} & {$[i: 1: 1]$} & & {$[-i: 1: 1]$} & {$[i: 1: 1]$} & \\
\hline$L_{2}$ & & & {$[-i:-1: 1]$} & {$[i:-1: 1]$} & {$[1: 0: 0]$} & {$[i:-1: 1]$} & {$[-i:-1: 1]$} & {$[1: 0: 0]$} \\
\hline$X_{3}$ & & & & {$[0: 1: 0]$} & {$[-i: 0: 1]$} & {$[-i: 1: 1]$} & {$[-i:-1: 1]$} & {$[0: 1: 0]$} \\
\hline$Y_{3}$ & & & & & {$[i: 0: 1]$} & {$[i:-1: 1]$} & {$[i: 1: 1]$} & {$[0: 1: 0]$} \\
\hline$Z_{3}$ & & & & & & {$[0: 0: 1]$} & {$[0: 0: 1]$} & {$[1: 0: 0]$} \\
\hline$X_{4}$ & & & & & & & {$[0: 0: 1]$} & {$[-i: 1: 0]$} \\
\hline$Y_{4}$ & & & & & & & & {$[i: 1: 0]$}
\end{tabular}

Table 1: The intersection data of the curves we track through the pencil.

Multiplicities greater than one are indicated by subscripts.

Now we will blow up at the intersection points of $T_{[1: 0]}$ and $T_{[0: 1]}$, and we would like to know how all of these curves intersect at those points and with what multiplicities, in order to determine their homology classes after blowing up the pencil. We summarize the relevant intersection data in Table 1.

To obtain an elliptic fibration, we blow up at the intersection points of $C_{1} \cup L_{1}$ with $C_{2} \cup L_{2}$. By Table 1, the relevant points are

$$
\begin{aligned}
& {[0: 0: 1]=C_{1} \cap C_{2} \cap Z_{3} \cap X_{4} \cap Y_{4}, \quad[0: 1: 0]=C_{1} \cap C_{2} \cap X_{3} \cap Y_{3} \cap Z_{4},} \\
& {[i: 1: 1]=L_{1} \cap C_{2} \cap Y_{3} \cap Y_{4} \text {, }} \\
& {[-i: 1: 1]=L_{1} \cap C_{2} \cap X_{3} \cap X_{4},} \\
& {[i:-1: 1]=C_{1} \cap L_{2} \cap Y_{3} \cap X_{4}, \quad[-i:-1: 1]=C_{1} \cap L_{2} \cap X_{3} \cap Y_{4}} \\
& {[1: 0: 0]=L_{1} \cap L_{2} \cap Z_{3} \cap Z_{4} \text {. }}
\end{aligned}
$$

Observe that $[0: 0: 1]$ and $[0: 1: 0]$ appear as intersection points of multiplicity two in $C_{1}, C_{2}, Z_{3}$ and $C_{1}, C_{2}, Z_{4}$ respectively, but all other intersections are transverse. We will need to blow up twice at the multiplicity two points, and once at each other point, to eliminate the base locus of the pencil.

We will denote the generator of $H_{2}\left(\mathbb{C P}^{2} ; \mathbb{Z}\right)$ by $h$. Note that the homology class represented by one of the listed curves is $h$ if the curve is linear, and $2 h$ if the curve is quadratic. 
Blow up, introducing the exceptional sphere $E_{1}$ (with homology class $e_{1}$ ), at $[0: 0: 1]$. Then the proper transforms $\widetilde{X}_{4}$ and $\widetilde{Y}_{4}$ intersect $E_{1}$ at distinct points, and $\widetilde{C}_{1}, \widetilde{C}_{2}$, and $\widetilde{Z}_{3}$ all intersect at a common third point on $E_{1}$. Note that while $X_{4} \cup Y_{4} \cup Z_{4}$ was a fiber of the Lefschetz pencil, the homology class $e_{1}$ appears with multiplicity two in its proper transform, while it appears with multiplicity one in the proper transforms of the other fibers. Therefore the Lefschetz pencil on the blown-up manifold now has a fiber $\tilde{X}_{4} \cup \tilde{Y}_{4} \cup \widetilde{Z}_{4} \cup E_{1}$ which includes the exceptional sphere with multiplicity one so that the fibers all represent the same homology class.

Next, we blow up at $\widetilde{C}_{1} \cap \widetilde{C}_{2} \cap \widetilde{Z}_{3} \cap E_{1}$, introducing a new exceptional sphere $E_{2}$ which intersects the proper transforms $\widetilde{C}_{1}, \widetilde{C}_{2}, \widetilde{Z}_{3}$, and $\widetilde{E}_{1}$. At this point the homology classes of all proper transforms are as follows:

$$
\begin{array}{llll}
{\left[\widetilde{C}_{1}\right]=2 h-e_{1}-e_{2},} & {\left[\tilde{L}_{1}\right]=h,} & & {\left[\widetilde{E}_{1}\right]=e_{1}-e_{2},} \\
{\left[\widetilde{C}_{2}\right]=2 h-e_{1}-e_{2},} & {\left[\tilde{L}_{2}\right]=h,} & & \\
{\left[\widetilde{X}_{3}\right]=h,} & {\left[\widetilde{Y}_{3}\right]=h,} & {\left[\widetilde{Z}_{3}\right]=h-e_{1}-e_{2},} \\
{\left[\tilde{X}_{4}\right]=h-e_{1},} & {\left[\tilde{Y}_{4}\right]=h-e_{1},} & {\left[\widetilde{Z}_{4}\right]=h .}
\end{array}
$$

Note that $\widetilde{E}_{1}$ is included with multiplicity one in a fiber with $\widetilde{X}_{4} \cup \widetilde{Y}_{4} \cup \widetilde{Z}_{4}$.

A similar situation occurs at $[0: 1: 0]$. We blow up two new exceptional spheres represented homology classes $e_{3}$ and $e_{4}$ at this point. This time we must include $\widetilde{E}_{3}$ with multiplicity one in a fiber with $\tilde{X}_{3} \cup \tilde{Y}_{3} \cup \widetilde{Z}_{3}$.

Finally we blow up once at the points $[i: 1: 1],[-i: 1: 1],[i:-1: 1],[-i:-1: 1]$, and $[1: 0: 0]$, introducing exceptional homology classes $e_{5}, e_{6}, e_{7}, e_{8}$, and $e_{9}$ respectively. The homology classes of the proper transforms of the relevant curves in the four singular fibers are given as in the statement of the proposition.

Finally, we construct an elliptic fibration with two $I_{5}$ fibers and two fishtails.

Lemma 4.3 There is an elliptic fibration on $E(1)=\mathbb{C P}^{2} \# 9 \overline{\mathbb{C P}^{2}}$ with two $I_{5}$ fibers two fishtails and a section. Both $I_{5}$ fibers are made up of five $-2-$ spheres $C_{1}, \ldots, C_{5}$, $D_{1}, \ldots, D_{5}$ with homology classes given below:

$$
\begin{array}{ll}
{\left[C_{1}\right]=e_{1}-e_{6},} & {\left[D_{1}\right]=e_{4}-e_{9},} \\
{\left[C_{2}\right]=h-e_{1}-e_{4}-e_{9},} & {\left[D_{2}\right]=h-e_{3}-e_{4}-e_{5},} \\
{\left[C_{3}\right]=h-e_{2}-e_{5}-e_{8},} & {\left[D_{3}\right]=e_{5}-e_{8},} \\
{\left[C_{4}\right]=e_{2}-e_{7},} & {\left[D_{4}\right]=h-e_{1}-e_{5}-e_{6},} \\
{\left[C_{5}\right]=h-e_{1}-e_{2}-e_{3},} & {\left[D_{5}\right]=h-e_{2}-e_{4}-e_{7} .}
\end{array}
$$




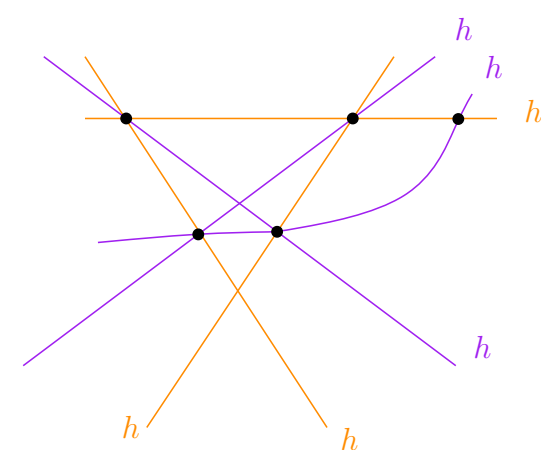

Figure 14: Initial configuration of lines.

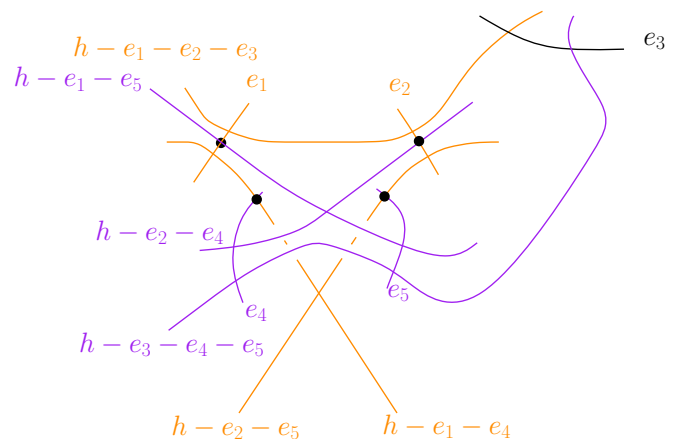

Figure 15: The pencil after first five blow-ups.

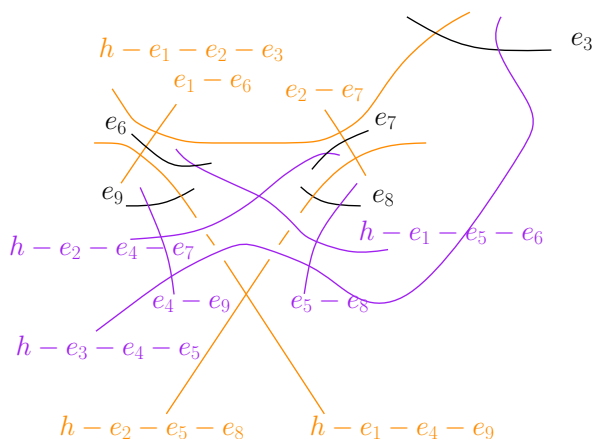

Figure 16: Elliptic fibration.

Proof Start with a configuration of six complex projective lines, intersecting as shown in Figure 14. We can view these lines as two degenerate cubic curves indicated by distinct colors in Figure 14, which generate a Lefschetz pencil on $\mathbb{C P}^{2}$.

Blowing up this pencil along the five intersection points between the orange and purple curves yields the configuration in Figure 15. In order to keep the homology classes of the two curves the same so they continue to define a pencil, two of the exceptional spheres must be included with multiplicity one in the orange curve, and two must be included with multiplicity one in the purple curve. The resulting curves defining a pencil on $\mathbb{C P}^{2} \# 5 \overline{\mathbb{C P}^{2}}$ intersect in four distinct points, and blowing up at each of these points yields an elliptic fibration shown in Figure 16.

The proper transforms of the original curves defining the pencil become the $I_{5}$ fibers representing the specified homology classes. Any other singular fibers can be perturbed to be generic nodal (fishtail) singular fibers, and an Euler characteristic computation indicates there are two of these. 


\section{Constructions of symplectic exotic 4-manifolds}

In this section we put together the information from the previous sections, to construct small exotic symplectic manifolds using star surgery. In the first construction, we will perform a full analysis of the smooth invariants of the resulting manifold. In the subsequent examples, we provide abridged computations that suffice to prove that the examples are exotic copies of $\mathbb{C} \mathrm{P}^{2} \# k \overline{\mathbb{C P}^{2}}$.

\section{A A symplectic exotic $\mathbb{C} P^{2} \# 8 \overline{\mathbb{C P}^{2}}$}

5A1 The construction Our first construction of an exotic $\mathbb{C P}^{2} \# 8 \overline{\mathbb{C P}^{2}}$ uses the elliptic fibration given in Lemma 4.1, which has an $I_{3}$ fiber and $I_{0}^{*}$ fiber, three fishtail fibers, and a section, with homology classes specified in the proof of the lemma.

Lemma 5.1 The configuration $\mathcal{S}_{2}$ symplectically embeds into $\mathbb{C} \mathrm{P}^{2} \# 11 \overline{\mathbb{C P}^{2}}$ such that its vertices represent the following homology classes, (where $\left.f=3 h-\left(e_{1}+\cdots+e_{9}\right)\right)$ :

$$
\begin{aligned}
& {\left[u_{0}\right]=2 f+e_{1}-2 e_{10}-2 e_{11},} \\
& {\left[u_{1}\right]=h-e_{1}-e_{2}-e_{3},} \\
& {\left[u_{2}\right]=h-e_{1}-e_{4}-e_{5},} \\
& {\left[u_{3}\right]=h-e_{1}-e_{6}-e_{7},} \\
& {\left[u_{4}\right]=h-e_{1}-e_{8}-e_{9} .}
\end{aligned}
$$

Proof Consider the elliptic fibration constructed in Lemma 4.1. In this fibration we see the following symplectic spheres:

- The components $S_{1}, \ldots, S_{5}$ of the $I_{0}^{*}$ fiber, with homology classes $\left[S_{1}\right]=$ $h-e_{1}-e_{2}-e_{3},\left[S_{2}\right]=h-e_{1}-e_{4}-e_{5},\left[S_{3}\right]=h-e_{1}-e_{6}-e_{7},\left[S_{4}\right]=e_{1}-e_{8}$ and $\left[S_{5}\right]=e_{8}-e_{9}$.

- The component $V_{1}$ of the $I_{3}$ fiber with homology class $\left[V_{1}\right]=h-e_{1}-e_{8}-e_{9}$.

- The exceptional sphere $E_{9}$ which is a section.

Take two fishtail fibers and blow up their double points. The proper transforms $F_{1}$ and $F_{2}$ are now symplectic spheres in $\mathbb{C P}^{2} \# 11 \overline{\mathbb{C P}^{2}}$. Their cohomology classes are $\left[F_{1}\right]=f-2 e_{10}$ and $\left[F_{2}\right]=f-2 e_{11}$.

Take the union of spheres $u_{0}=F_{1} \cup F_{2} \cup E_{9} \cup S_{4} \cup S_{5}$. After symplectically smoothing its double points, $u_{0}$ gives a symplectic sphere whose homology class is $\left[u_{0}\right]=2 f+e_{1}-2 e_{10}-2 e_{11}$. Let $u_{1}=S_{1}, u_{2}=S_{2}, u_{3}=S_{3}, u_{4}=V_{1}$. Then the union $\bigcup_{i=0}^{4} u_{i}$ gives the required embedding of $\mathcal{S}_{2}$. 


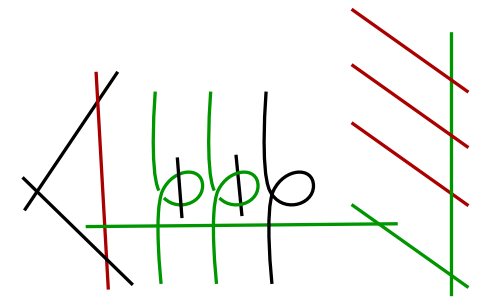

Figure 17: A blow-up of the elliptic fibration of Lemma 4.1 shown in Figure 12. The symplectic resolution of the union of the green curves is the sphere $u_{0}$, and the four red curves are the spheres $u_{1}, \ldots, u_{4}$.

Using this embedding of $\mathcal{S}_{2}$, perform star surgery on $\mathbb{C P}^{2} \# 11 \overline{\mathbb{C} P^{2}}$ by cutting out this embedded $\mathcal{S}_{2}$ and replacing it with $\mathcal{T}_{2}$ resulting in a manifold

$$
X=\left(\left(\mathbb{C P}^{2} \# 11 \overline{\mathbb{C P}^{2}}\right) \backslash \mathcal{S}_{2}\right) \cup_{\partial} \mathcal{T}_{2} .
$$

Lemma 5.2 The manifold $X$ is homeomorphic to $\mathbb{C P}^{2} \# 8 \overline{\mathbb{C P}^{2}}$.

Proof First we show the manifold $X$ is simply connected. By Proposition 3.1, it suffices to show that the dual circle of one of the -2 -spheres on $\partial \mathcal{S}_{2}$ bounds a disk in $\mathbb{C P}^{2} \# 11 \overline{\mathbb{C P}^{2}} \backslash \mathcal{S}_{2}$. By our construction the sphere $u_{4}$ is a part of the $I_{3}$ fiber. Hence the meridian of $u_{4}$ bounds a disk $D$ in the complement of $\mathcal{S}_{2}$ which is contained in a sphere component of the $I_{3}$ fiber transversely intersecting $u_{4}$.

Next we show that the intersection form of $X$ is isomorphic to the intersection form of $\mathbb{C P}^{2} \# 8 \overline{\mathbb{C P}^{2}}$. We calculate the Euler characteristic and signature to get

$$
\begin{aligned}
& \chi(X)=\chi\left(\mathbb{C P}^{2} \# 11 \overline{\mathbb{C P}^{2}}\right)-\chi\left(\mathcal{S}_{2}\right)+\chi\left(\mathcal{T}_{2}\right)=11, \\
& \sigma(X)=\sigma\left(\mathbb{C P}^{2} \# 11 \overline{\mathbb{C P}^{2}}\right)-\sigma\left(\mathcal{S}_{2}\right)+\sigma\left(\mathcal{T}_{2}\right)=-7 \text {. }
\end{aligned}
$$

These imply that $b_{2}^{+}(X)=1$ and $b_{2}(X)=9$. The intersection form cannot be even, otherwise it would be written as a direct sum of hyperbolic pieces and $E_{8} \mathrm{~s}$, but the values of $b_{2}^{+}(X)$ and $b_{2}(X)$ say that such a decomposition is impossible. Hence $X$ is homeomorphic to $\mathbb{C P}^{2} \# 8 \overline{\mathbb{C P}^{2}}$ by Freedman's theorem [19].

5A2 Kodaira dimension In this section we compute the symplectic Kodaira dimension [27] of $X$. Along the way, we distinguish $X$ from $\mathbb{C P}^{2} \# 8 \overline{\mathbb{C P}^{2}}$. Our argument is similar to Park's [36]. 
Definition 5.3 [27] For a minimal symplectic 4-manifold $(M, \omega)$ with symplectic canonical class $K_{\omega}$, the Kodaira dimension of $(M, \omega)$ is defined in the following way:

$$
\kappa(M, \omega)= \begin{cases}-\infty & \text { if } K_{\omega} \cdot[\omega]<0 \text { or } K_{\omega} \cdot K_{\omega}<0, \\ 0 & \text { if } K_{\omega} \cdot[\omega]=0 \text { and } K_{\omega} \cdot K_{\omega}=0, \\ 1 & \text { if } K_{\omega} \cdot[\omega]>0 \text { and } K_{\omega} \cdot K_{\omega}=0, \\ 2 & \text { if } K_{\omega} \cdot[\omega]>0 \text { and } K_{\omega} \cdot K_{\omega}>0 .\end{cases}
$$

The Kodaira dimension of a nonminimal manifold is defined to be that of any of its symplectic minimal models.

It is known that the Kodaira dimension is well-defined for every closed symplectic 4-manifold and depends only on the diffeomorphism type of the manifold.

Let $K$ and $\omega$ denote the canonical class and the symplectic class of $\mathbb{C P}^{2} \# 11 \overline{\mathrm{CP}^{2}}$ respectively. Let $X_{0}=\mathbb{C P}^{2} \# 11 \overline{\mathbb{C P}^{2}} \backslash \mathcal{S}_{2}$. Then

$$
\begin{aligned}
& K=\left.K\right|_{X_{0}}+\left.K\right|_{\mathcal{S}_{2}}, \\
& \omega=\left.\omega\right|_{X_{0}}+\left.\omega\right|_{\mathcal{S}_{2}} .
\end{aligned}
$$

Hence

$$
K \cdot \omega=\left.\left.K\right|_{X_{0}} \cdot \omega\right|_{X_{0}}+\left.\left.K\right|_{\mathcal{S}_{2}} \cdot \omega\right|_{\mathcal{S}_{2}} .
$$

Let $K_{X}$, and $\omega_{X}$ denote the canonical class and the symplectic class of $X$ respectively. Then

$$
\begin{aligned}
& K_{X}=\left.K\right|_{X_{0}}+K_{\mathcal{T}_{2}}, \\
& \omega_{X}=\left.\omega\right|_{X_{0}}+\left.\omega\right|_{\mathcal{T}_{2}} .
\end{aligned}
$$

Since $K_{\mathcal{T}_{2}}=0$ by Proposition 3.3, we have

$$
K_{X} \cdot \omega_{X}=\left.\left.K\right|_{X_{0}} \cdot \omega\right|_{X_{0}}=K \cdot \omega-\left.\left.K\right|_{\mathcal{S}_{2}} \cdot \omega\right|_{\mathcal{S}_{2}} .
$$

Lemma 5.4 For every $k>0$, the manifold $\mathbb{C P}^{2} \# k \overline{\mathbb{C P}^{2}}$ admits a symplectic structure whose cohomology class is given by $\omega=a h-b_{1} e_{1}-\cdots-b_{k} e_{k}$ for some rational numbers $a, b_{1}, \ldots, b_{k}$ with $a>b_{1}>\cdots>b_{k}$ and $a>b_{1}+\cdots+b_{k}$.

Proof Note that $a$ is the symplectic area of $\mathbb{C P}^{1} \subset \mathbb{C} \mathrm{P}^{2}$ and $b_{j}$ is the symplectic area of the exceptional sphere $e_{j}$ for all $j=1, \ldots, k$. By [29, Chapter 7], we have $b_{j}=\lambda^{2}$ where $\lambda$ is the weight of the corresponding blow-up. In other words $\lambda$ is the radius of the Darboux ball which will be removed during the blow-up process. Since the weights of the blow-ups can be chosen to be arbitrarily small, the result follows. 
Lemma 5.5

$$
K_{X} \cdot \omega_{X}>0
$$

Proof Write

$$
\begin{aligned}
& K=-3 h+e_{1}+\cdots+e_{11}, \\
& \omega=a h-b_{1} e_{1}-\cdots-b_{11} e_{11},
\end{aligned}
$$

where $a, b_{1}, \ldots, b_{11}$ are rational numbers such that $a>b_{1}>\cdots>b_{11}$ and

$$
a>b_{1}+\cdots+b_{11} .
$$

Then we have

$$
K \cdot \omega=-3 a+b_{1}+\cdots+b_{11} .
$$

Let $\gamma_{0}, \ldots, \gamma_{4}$ be the basis of $H^{2}\left(\mathcal{S}_{2}, \mathbb{Q}\right)$ which is dual to $u_{0}, \ldots, u_{4}$. Then the adjunction formula implies

$$
\left.K\right|_{\mathcal{S}_{2}}=\left(K \cdot u_{0}\right) \gamma_{0}+\cdots+\left(K \cdot u_{4}\right) \gamma_{4}=3 \gamma_{0} .
$$

We calculate the restriction of the symplectic class on $\mathcal{S}_{2}$ using Lemma 5.1:

$$
\begin{aligned}
\left.\omega\right|_{\mathcal{S}_{2}}= & \left(\omega \cdot u_{0}\right) \gamma_{0}+\cdots+\left(\omega \cdot u_{4}\right) \gamma_{4} \\
= & \left(6 a-b_{1}-2 b_{2}-\cdots-2 b_{11}\right) \gamma_{0}+\left(a-b_{1}-b_{2}-b_{3}\right) \gamma_{1} \\
& \quad+\left(a-b_{1}-b_{4}-b_{5}\right) \gamma_{2}+\left(a-b_{1}-b_{6}-b_{7}\right) \gamma_{3}+\left(a-b_{1}-b_{8}-b_{9}\right) \gamma_{4} .
\end{aligned}
$$

Let $P$ denote the intersection matrix for $\mathcal{S}_{2}$. Then

$$
P^{-1}=-\frac{1}{12}\left(\begin{array}{lllll}
4 & 2 & 2 & 2 & 2 \\
2 & 7 & 1 & 1 & 1 \\
2 & 1 & 7 & 1 & 1 \\
2 & 1 & 1 & 7 & 1 \\
2 & 1 & 1 & 1 & 7
\end{array}\right) \text {. }
$$

Hence

$$
\begin{aligned}
\left.\left.K\right|_{\mathcal{S}_{2}} \cdot \omega\right|_{\mathcal{S}_{2}=}= & \frac{1}{4}\left[4\left(6 a-b_{1}-2 b_{2}-\cdots-2 b_{11}\right)+2\left(a-b_{1}-b_{2}-b_{3}\right)\right. \\
& \left.+2\left(a-b_{1}-b_{4}-b_{5}\right)+2\left(a-b_{1}-b_{6}-b_{7}\right)+2\left(a-b_{1}-b_{8}-b_{9}\right)\right] \\
=- & \frac{1}{4}\left[32 a-12 b_{1}-10 b_{2}-10 b_{3}-\cdots-10 b_{9}-8 b_{10}-8 b_{11}\right] .
\end{aligned}
$$

Therefore

$$
\begin{aligned}
K_{X} \cdot \omega_{X} & =\left.\left.K\right|_{X_{0}} \cdot \omega\right|_{X_{0}}=K \cdot \omega-\left.\left.K\right|_{\mathcal{S}_{2}} \cdot \omega\right|_{\mathcal{S}_{2}} \\
& =-3 a+b_{1}+\cdots+b_{11}+\left[8 a-3 b_{1}-\frac{5}{2} b_{2}-\cdots-\frac{5}{2} b_{9}-2 b_{10}-2 b_{11}\right] \\
& =5 a-2 b_{1}-\frac{3}{2} b_{2}-\cdots-\frac{3}{2} b_{9}-b_{10}-b_{11} \\
& >0 \quad \text { (by Equation (5-1)). }
\end{aligned}
$$


Remark 5.6 The important input which ensures there is a symplectic form on $X$ with $K_{X} \cdot \omega_{X}>0$ is the high multiplicity of the homology class $h$ in the classes $\left[u_{i}\right]$ of the spheres in the embedding of $\mathcal{S}_{2}$. Note that if the embedding of $\mathcal{S}_{2}$ were completely disjoint from the $\mathbb{C P}^{1}$ representing $h$, then by McDuff's theorem, the resulting star surgered manifold would be a standard blow-up of $\mathbb{C P}^{2}$. This computation provides a quantitative way of ensuring that when the plumbing spheres intersect $\mathbb{C P}^{1}$ enough for the star surgery to produce an exotic smooth structure.

Proposition 5.7 The manifold $X$ is not diffeomorphic to $\mathbb{C P}^{2} \# 8 \overline{\mathrm{CP}^{2}}$.

Proof The standard symplectic form on $\mathbb{C P}^{2} \# k \overline{\mathbb{C P}^{2}}$ satisfies $K \cdot \omega<0$. According to [28, Theorem $\mathrm{D}]$, there is a unique symplectic structure on $\mathbb{C P}^{2} \# k \overline{\mathbb{C} \mathrm{P}^{2}}$ for $2 \leq k \leq 9$ up to diffeomorphism and deformation. Hence $\mathbb{C P}^{2} \# 8 \overline{\mathbb{C P}^{2}}$ does not admit a symplectic structure with $K \cdot \omega>0$. We have just seen that $K_{X} \cdot \omega_{X}>0$. Hence $X$ is not diffeomorphic to $\mathbb{C P}^{2} \# 8 \overline{\mathbb{C P}^{2}}$.

Proposition 5.8 The manifold $X$ is minimal.

The proof is postponed until the next section. With this in hand, we are ready to determine the Kodaira dimension of $X$.

Proposition 5.9 The manifold $X$ has symplectic Kodaira dimension 2 .

Proof Recall that the symplectic Kodaira dimension is defined on the minimal model. Since $X$ is minimal, $K_{X} \cdot \omega_{X}>0$, and $K_{X}^{2}=3 \sigma(X)+2 \chi(X)=1>0$, its symplectic Kodaira dimension is 2 .

This proposition shows that $X$ can also be distinguished from $\mathbb{C P}^{2} \# 8 \overline{\mathbb{C P}^{2}}$ using the symplectic Kodaira dimension, since $\kappa\left(\mathbb{C P ^ { 2 }} \# 8 \overline{\mathbb{C P}^{2}}\right)=\kappa\left(\mathbb{C P} P^{2}\right)=-\infty$.

5A3 Minimality Throughout this section, we assume that the reader is familiar with the Seiberg-Witten invariants of manifolds with $b_{2}^{+}=1$. See $[31 ; 17 ; 42]$ for excellent expositions.

In order to prove the minimality of $X$ one needs to know the effect of star surgery on Seiberg-Witten invariants. It suffices to know all the Seiberg-Witten basic classes of $X$. The effect of such cut and paste operations on Seiberg-Witten invariants was studied by Michalogiorgaki in [30] in a more general framework; see also [39] for an analogous result from the perspective of Heegaard Floer theory. 
Theorem 5.10 [30, Theorem 1] Suppose $Y$ is a rational homology sphere which is a monopole $L$-space. Let $P$ and $B$ be negative-definite 4-manifolds with $b_{1}(P)=$ $b_{1}(B)=0$ and $\partial P=\partial B=Y$. Let $X=Z \bigcup_{Y} P$ and $X^{\prime}=Z \bigcup_{Y} B$, for some $4-$ manifold $Z$. If $\mathfrak{s} \in \operatorname{Spin}^{c}(X), \mathfrak{s}^{\prime} \in \operatorname{Spin}^{c}\left(X^{\prime}\right), d_{X}(\mathfrak{s}), d_{X^{\prime}}\left(\mathfrak{s}^{\prime}\right) \geq 0$ and $\mathfrak{s}\left|Z=\mathfrak{s}^{\prime}\right|_{Z}$, then $\mathrm{SW}_{X}(\mathfrak{s})=\mathrm{SW}_{X^{\prime}}\left(\mathfrak{s}^{\prime}\right)$.

In the case that $b_{2}^{+}(X)=1, \mathrm{SW}_{X, a_{1}}(\mathfrak{s})=\mathrm{SW}_{X^{\prime}, a_{2}}\left(\mathfrak{s}^{\prime}\right)$, where $a_{1} \in H_{2}(X, \mathbb{Z})$, $a_{2} \in H_{2}\left(X^{\prime}, \mathbb{Z}\right)$ specify chambers such that $\left.a_{1}\right|_{P}=\left.a_{2}\right|_{B}=0$ and $\left.a_{1}\right|_{Z}=\left.a_{2}\right|_{Z}$.

This result and the wall-crossing formula reduces the problem of determining basic classes of $X$ to a cohomology computation. In this section, we incorporate a search method invented by Ozsváth and Szabó to find all the basic classes of $X$. First we find a homology class that determines a common chamber for the manifolds before and after star surgery.

Lemma 5.11 The element $V \in H_{2}\left(\mathbb{C P}^{2} \# 11 \overline{\mathbb{C P}^{2}}\right)$ defined by $V=86 h-36 e_{1}-25 e_{2}-25 e_{3}-25 e_{4}-25 e_{5}-25 e_{6}-25 e_{7}-19 e_{8}-31 e_{9}-20 e_{10}-20 e_{11}$ satisfies the following conditions:

(1) $V \cdot\left[u_{i}\right]=0$ for $i=0, \ldots, 4$ (ie $V$ is orthogonal to each embedded sphere of $\mathcal{S}_{2}$ ),

(2) $V \cdot V>0$,

(3) $V \cdot h>0$,

(4) $V \cdot K>0$, where $K=-3 h+e_{1}+\cdots+e_{11}$ is the canonical class of $\mathbb{C P}^{2} \# 11 \overline{\mathbb{C P}^{2}}$.

Proof The proof is a direct computation.

Lemma 5.12 The small perturbation Seiberg-Witten invariant of $X$ at the canonical class $\widetilde{K} \in H^{2}(X, \mathbb{Z})$ is nonzero.

Proof We first compute the Seiberg-Witten invariant of $\mathbb{C P} P^{2} \# 11 \overline{\mathbb{C} P^{2}}$ in the chamber determined by the element $V$ in Lemma 5.11. Orient $H_{2}^{+}\left(\mathbb{C P}^{2} \# 11 \overline{\mathbb{C P}^{2}}\right)$ with $h$. Note that the homology class $h$ gives the chamber of the positive scalar curvature metric hence all Seiberg-Witten invariants of $\mathbb{C P}^{2} \# 11 \overline{\mathbb{C P}^{2}}$ are zero in this chamber. In particular $\mathrm{SW}_{\mathbb{C P}^{2} \# 11 \overline{\mathbb{C P}^{2}}, h}(K)=0$ for the canonical class $K$. By part (2) of Lemma 5.11, $V \in H_{2}^{+}\left(\mathbb{C P}^{2} \# 11 \overline{\mathbb{C P}^{2}}\right)$, and by part (3) $V$ has the correct orientation. Hence $V$ determines a chamber. Since $K \cdot h<0$, part (4) says that there is a wall between the chambers of $h$ and $V$ with respect to the canonical class. By the wall-crossing formula [28], $\mathrm{SW}_{\mathbb{C P}^{2} \# 11 \overline{\mathbb{C P}^{2}}, V}(K)= \pm 1$.

Next we relate the Seiberg-Witten invariants of $\mathbb{C P}^{2} \# 11 \overline{\mathbb{C P}^{2}}$ and $X$. The canonical class $\tilde{K}$ of $X$ satisfies $\widetilde{K}_{X \backslash \mathcal{T}_{2}}=K_{\mathbb{C P}^{2} \# 11 \overline{\mathbb{C P}^{2}} \backslash \mathcal{S}_{2}}$. Part (1) says $V$ determines a cham- 
ber in $X$. If we can show that $\partial \mathcal{T}_{2}$ is a monopole $L$-space then by Theorem 5.10, noting that $d_{X}(\tilde{K})=d_{\mathbb{C P}^{2} \# 11 \overline{\mathbb{C P}^{2}}}(K)=0$, we have $\operatorname{SW}_{\mathbb{C P}^{2} \# 11 \overline{\mathbb{C P}^{2}, V}}(K)=\operatorname{SW}_{X, V}(\tilde{K})$. Since there is a unique small perturbation chamber for manifolds with $b_{2}^{-} \leq 9$, the latter is equal to $\operatorname{SW}_{X}(\tilde{K})$.

It remains to see that $\partial \mathcal{T}_{2}=\partial \mathcal{S}_{2}$ is a monopole $L$-space. In fact this holds for $\partial \mathcal{S}_{i}$ for all $i$. The plumbing graph of $\mathcal{S}_{i}$ satisfies $|m(v)|>d(v)$ at each vertex $v$, where $m(v)$ is the weight of $v$ and $d(v)$ is the number of edges connected to $v$. In [33, Theorem 7.1], Ozsváth and Szabó show that the boundary of such a plumbing is a Heegaard Floer $L$-space. Their proof uses only the surgery exact triangle and the formal properties of Heegaard Floer homology. Hence it can be repeated to show that boundaries of such plumbings are also monopole $L$-spaces. Alternatively, one can refer to the recently established equivalence of the Heegaard Floer homology and the monopole Floer homology to see that every Heegaard Floer $L$-space is also a monopole $L$-space [26; 8].

Remark 5.13 Lemma 5.12 gives an alternative proof of Proposition 5.7. Indeed, the small perturbation Seiberg-Witten invariant is well defined for manifolds with $b_{2}^{-} \leq 9$ since there is a unique chamber for such manifolds. For $\mathbb{C P}^{2} \# k \overline{\mathbb{C P}^{2}}$ with $k \leq 9$ the unique chamber is the one given by the positive scalar curvature metric. Hence

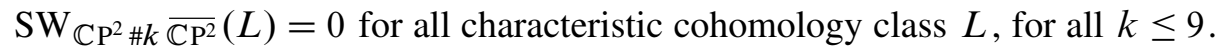

Proof of Proposition 5.8 By the blow-up formula for the Seiberg-Witten invariant, it suffices to show that the only basic classes of $X$ are $\pm \widetilde{K}$. Therefore, we want to check which integral characteristic cohomology classes (representing $\operatorname{Spin}^{c}$ structures) on $X$ are Seiberg-Witten basic classes. While we will show how to use Theorem 5.10 and the wall-crossing formula to compute the Seiberg-Witten invariant on a given cohomology class, there are infinitely many classes to check. The strategy of Ozsváth and Szabó (which they used to prove minimality of a rationally blown-down manifold) is to check only the finitely many adjunctive classes and then use the information about the adjunctive basic classes to rule out the possibility of nonadjunctive basic classes.

The fact that the homology of $\mathcal{T}_{2}$ has nontrivial rank makes the search somewhat more complicated than the case of rational blow-down. Even so, the computations can be handled by a simple computer program. First we find a basis for the subspace $\mathrm{H}_{2}\left(\mathbb{C P}^{2} \# 11 \overline{\mathbb{C P}^{2}}\right)$ which is orthogonal to the homology of the configuration $\mathcal{S}_{2}$. The following elements form such a basis:

$$
\begin{array}{ll}
A_{1}=h-e_{3}-e_{5}-e_{7}-e_{9}+e_{11}, & A_{2}:=-3 h+2 e_{1}+e_{3}+e_{5}+e_{7}+e_{9}+2 e_{10}+2 e_{11}, \\
A_{3}=e_{2}-e_{3}, \quad A_{4}=e_{4}-e_{5}, & A_{5}=e_{6}-e_{7}, \quad A_{6}=e_{10}-e_{11}, \quad A_{7}=e_{8}-e_{9} .
\end{array}
$$


Note that all of these homology classes can be represented by embedded spheres, excepting $A_{2}$ which can be represented by an embedded torus. Moreover, all of these surfaces can be chosen in the complement of $\mathcal{S}_{2}$ in $\mathbb{C P}^{2} \# 11 \overline{\mathbb{C P}^{2}}$. Additionally we let $A_{8}$ and $A_{9}$ be the homology generators of $\mathcal{T}_{2}$, which can be represented by embedded tori in $\mathcal{T}_{2}$, as indicated in Proposition 3.2. Then $A_{1}, \ldots, A_{9}$ represent a basis for $H_{2}(X ; \mathbb{Z})$ so we can represent cohomology classes in $H^{2}(X ; \mathbb{Z})$ by a tuple of integers representing $\left[\left\langle L, A_{1}\right\rangle, \ldots,\left\langle L, A_{9}\right\rangle\right]$.

First we will determine which of these tuples represent integral, characteristic, and adjunctive cohomology classes. Because the inverse of the intersection matrix has rational coefficients, some tuples of integers could represent rational, but not integral, homology classes. We check a mod 2 equivalence to see if the cohomology class is characteristic, and an adjunctive inequality for each $A_{i}$. Computationally, we find that there are exactly 243000 cohomology classes $L \in H^{2}(X, \mathbb{Q})$ which satisfy these conditions for all $i=1, \ldots, 9$ :

$$
\left\langle L, A_{i}\right\rangle \in \mathbb{Z}, \quad\left\langle L, A_{i}\right\rangle \equiv A_{i}^{2} \quad \bmod 2, \quad\left|\left\langle L, A_{i}\right\rangle\right| \leq-A_{i}^{2} .
$$

In order to test whether a characteristic class is basic for $X$, we first check whether the expected dimension is nonnegative and even (necessary conditions for the SeibergWitten invariant to be nonzero). Let $d_{X}(L):=\left(L^{2}-3 \sigma(X)-2 \chi(X)\right) / 4=\left(L^{2}-1\right) / 4$. In the second round of our search, we check how many of these cohomology classes satisfy $d_{X}(L) \in \mathbb{Z}, d_{X}(L) \geq 0$ and $d_{X}(L) \equiv 0 \bmod 2$. It turns out that there are 25040 such classes.

We will use Theorem 5.10 and the wall-crossing formula to calculate the Seiberg-Witten invariant evaluated on each of these 25040 classes. To do this, we must relate each class on $X$ to a class on $\mathbb{C P}^{2} \# 11 \overline{\mathbb{C P}^{2}}$ so that the two classes have equal restrictions to $X \backslash \mathcal{T}_{2}=\left(\mathbb{C} \mathrm{P}^{2} \# 11 \overline{\mathbb{C P}^{2}}\right) \backslash \mathcal{S}_{2}$. The difficulty is, once we restrict the chosen class to $X \backslash \mathcal{T}_{2}$, we must find a class on $\mathcal{S}_{2}$ which extends it over $\mathbb{C P}^{2} \# 11 \overline{\mathbb{C P}^{2}}$. For this, we compare the classes on $\partial \mathcal{S}_{2}$ which occur as the restriction of a class on $\mathcal{S}_{2}$ to the classes on $\partial \mathcal{T}_{2}=\partial \mathcal{S}_{2}$ that occur as the restriction of a class on $\mathcal{T}_{2}$. We showed that the image of the map $H^{2}\left(\mathcal{T}_{2}, \mathbb{Z}\right) \rightarrow H^{2}\left(\partial \mathcal{T}_{2}, \mathbb{Z}\right)$ is an index-2 subgroup, hence it has order 24 . Our aim is to find a numerical criterion for a characteristic cohomology class $L \in H^{2}\left(\mathcal{S}_{2}, \mathbb{Z}\right)$ to extend to $\mathcal{T}_{2}$ when restricted to $\partial \mathcal{S}_{2}=\partial \mathcal{T}_{2}$. First observe that the set of $\operatorname{Spin}^{c}$ structures on $\partial \mathcal{S}_{2}$ as a $H^{2}\left(\partial \mathcal{S}_{2}, \mathbb{Z}\right)$ torsor is isomorphic to $2 H^{2}\left(\mathcal{S}_{2}, \partial \mathcal{S}_{2}, \mathbb{Z}\right)$-orbits of characteristic elements in $H^{2}\left(\mathcal{S}_{2}, \mathbb{Z}\right)$. Hence two characteristic classes $L_{1}, L_{2} \in$ $H^{2}\left(\mathcal{S}_{2}, \mathbb{Z}\right)$ restrict to the same $\operatorname{Spin}^{c}$ structure on the boundary if and only if $L_{1}=L_{2}+$ $2 \operatorname{PD}(Z)$ for some $Z \in H_{2}\left(\mathcal{S}_{2}, \mathbb{Z}\right)$. We will use $d$-invariants to determine which orbits in $H^{2}\left(\mathcal{S}_{2}, \mathbb{Z}\right)$ restrict to a $\operatorname{Spin}^{c}$ structure on $\partial \mathcal{S}_{2}=\partial \mathcal{T}_{2}$ which appears in the boundary 
of a $\operatorname{Spin}^{c}$ structure on $\mathcal{T}_{2}$ and to choose distinguished representatives of these orbits which maximize the expected dimension of the Seiberg-Witten moduli space. Define $d_{\mathcal{S}_{2}}(L)=\left(L^{2}-3 \sigma\left(\mathcal{S}_{2}\right)-2 \chi\left(\mathcal{S}_{2}\right)\right) / 4=\left(L^{2}+3\right) / 4$. Observe that the $d_{\mathcal{S}_{2}}(L) \bmod 2$ is constant in the orbit $L+2 H^{2}\left(\mathcal{S}_{2}, \partial \mathcal{S}_{2}, \mathbb{Z}\right)$. Each orbit has a representative $L^{\prime}$ satisfying $\left[u_{i}\right]^{2}+2 \leq\left\langle L^{\prime},\left[u_{i}\right]\right\rangle \leq-\left[u_{i}\right]^{2}$ for all $i=0, \ldots, 4$, where each $u_{i}$ is a sphere appearing as a vertex in the star-shaped plumbing graph of $\mathcal{S}_{2}$. Computing $d_{\mathcal{S}_{2}}$ for all of these representatives, we see that the possible mod 2 reductions of $d_{\mathcal{S}_{2}}$ for characteristic cohomology classes on $\mathcal{S}_{2}$ are $\left\{\frac{-2}{3}, \frac{-1}{3}, \frac{-1}{4}, \frac{-1}{12}, 0, \frac{1}{4}, \frac{2}{3}, \frac{-11}{12}, 1\right\}$.

In the proof of Proposition 3.2, we computed the intersection form of $\mathcal{T}_{2}$. From this, we see that the possible mod 2 reductions of $d_{\mathcal{T}_{2}}$ for characteristic cohomology classes on $\mathcal{T}_{2}$ are $\left\{\frac{-1}{3}, 0, \frac{2}{3}, 1\right\}$. We observe that exactly 24 of the $2 H^{2}\left(\mathcal{S}_{2}, \partial \mathcal{S}_{2}, \mathbb{Z}\right)$ orbits have the mod 2 reductions of their $d_{\mathcal{S}_{2}}$ in this set. Hence we conclude that a characteristic cohomology class $L \in H^{2}\left(\mathcal{S}_{2}, \mathbb{Z}\right)$ extends to $\mathcal{T}_{2}$ if and only if $d_{\mathcal{S}_{2}}(L) \in$ $\left\{\frac{-1}{3}, 0, \frac{2}{3}, 1\right\} \bmod 2$. We can explicitly write distinguished representatives of these 24 orbits. They are the elements of the following set:

$$
\begin{aligned}
\Phi=\{ & {[1,0,0,0,0],[-3,2,2,2,2],[-1,2,0,0,2],[-3,0,2,2,0], } \\
& {[1,2,0,2,0],[3,0,0,0,0],[-1,2,2,0,0],[-3,2,0,2,0],[-1,0,2,0,2], } \\
& {[-3,0,0,2,2],[1,0,2,2,0],[1,0,0,2,2],[-1,0,2,2,0],[-3,0,2,0,2], } \\
& {[-3,0,0,0,0],[1,2,0,0,2],[5,0,0,0,0],[-3,2,0,0,2],[-1,0,0,2,2], } \\
& {[1,0,2,0,2],[-1,2,0,2,0],[-3,2,2,0,0],[1,2,2,0,0],[-1,0,0,0,0]\} . }
\end{aligned}
$$

Here each cohomology class $L \in H^{2}\left(\mathcal{S}_{2}, \mathbb{Z}\right)$ is represented by the tuple $\left[\left\langle L,\left[u_{0}\right]\right\rangle, \ldots\right.$, $\left.\left\langle L,\left[u_{4}\right]\right\rangle\right]$. Elements of $\Phi$ maximize $d_{\mathcal{S}_{2}}$ in their respective $2 H^{2}\left(\mathcal{S}_{2}, \partial \mathcal{S}_{2}, \mathbb{Z}\right)$ orbits.

We continue our search for basic classes of $X$. Recall that in round two, we got 25, 040 potential adjunctive basic classes. We restrict each one to $X \backslash \mathcal{T}_{2}$ (ie we forget the intersections with $A_{8}$ and $A_{9}$ ). Each of these characteristic cohomology classes glues to exactly one element of the set $\Phi$ to define a characteristic cohomology class on $\mathbb{C P}^{2} \# 11 \overline{\mathbb{C P}^{2}}$. There are 600,960 triples $(A, B, C)$ where $A$ is an adjunctive class on $\mathcal{T}_{2}, B$ is an adjunctive class on $X \backslash \mathcal{T}_{2}=\mathbb{C P}^{2} \# 11 \overline{\mathbb{C P}^{2}} \backslash \mathcal{S}_{2}, C$ is one of the 24 distinguished cohomology classes on $\mathcal{S}_{2}$, and the pair $(B, A)$ is one of the 25,040 potential adjunctive basic classes on $X$. We now restrict our attention to all triples where the combination $(B, C)$ represents a cohomology class on $\mathbb{C P}^{2} \# 11 \overline{\mathbb{C P}^{2}}$ with $d \in \mathbb{Z}, d \geq 0$ and $d \equiv 2 \bmod 2$. This leaves us with 219,064 possible triples. In only 122,212 of these triples does $(B, C)$ represent an integral and characteristic cohomology class on $\mathbb{C P}^{2} \# 11 \overline{\mathbb{C P}^{2}}$. Finally, we check whether the chamber determined by $V$ in $\mathbb{C P}^{2} \# 11 \overline{\mathbb{C P}^{2}}$ is the same as the chamber of the positive scalar curvature metric with respect to each of the characteristic cohomology classes $(B, C)$ in the remaining 
triples by comparing the signs of $(B, C) \cdot V$ with $(B, C) \cdot H$. When the chambers agree, $B$ will not descend to a basic class on $X$, but when they disagree the wall-crossing formula and Theorem 5.10 ensure that $(A, B)$ is a basic class on $X$. It turns out that only two of the remaining triples $(A, B, C)$ have the property that $V$ is in a different chamber than $H$ with respect to $(B, C)$. These are necessarily the canonical class and its negative. Hence $\widetilde{K}$ and $-\widetilde{K}$ are the only adjunctive basic classes of $X$.

Lastly we argue that there can be no nonadjunctive basic class. If there was such an $L$, then the adjunction relations [32] imply that adding or subtracting twice the Poincaré dual of any surface with negative self-intersection where the adjunction inequality fails, we would obtain another basic class $L^{\prime}$ with $d_{X}\left(L^{\prime}\right)>d_{X}(L)$.

Since $X$ has only finitely many basic classes this process eventually stops at an adjunctive basic class, $\bar{L}$ with $d_{X}(\bar{L})>d_{X}(L) \geq 0$, but this is a contradiction because $\tilde{K}$ and $-\tilde{K}$ are the only adjunctive basic classes and $d_{X}(\tilde{K})=d_{X}(-\tilde{K})=0$.

Proof of Theorem 1.1 Our theorem follows from Lemma 5.2, Proposition 5.7, Proposition 5.8, and Proposition 5.9.

Remark 5.14 The Seiberg-Witten invariants of $X$ agree up to isomorphism with all known examples of minimal exotic $\mathbb{C P}^{2} \# 8 \overline{\mathbb{C P}^{2}}[36 ; 42 ; 16 ; 30]$. Hence we cannot distinguish $X$ from these manifolds using Seiberg-Witten invariants.

\section{B An exotic $\mathbb{C P}^{2} \# 7 \overline{\mathbb{C P}^{2}}$}

Lemma 5.15 There is an embedding of the star-shaped plumbing $\mathcal{Q}$ into $\mathbb{C P}^{2} \# 12 \overline{\mathbb{C P}^{2}}$ such that the spheres represent the following homology classes:

$$
\begin{aligned}
{\left[u_{0}\right] } & =6 h-2 e_{1}-e_{2}-2 e_{3}-\cdots-2 e_{9}-2 e_{11}-2 e_{12}, \\
{\left[u_{1,1}\right] } & =2 h-e_{1}-e_{2}-e_{3}-e_{4}-e_{5}-e_{6}, \\
{\left[u_{2,1}\right] } & =h-e_{1}-e_{2}-e_{9} \\
{\left[u_{2,2}\right] } & =h-e_{3}-e_{5}-e_{7}, \\
{\left[u_{3,1}\right] } & =h-e_{1}-e_{6}-e_{7} \\
{\left[u_{3,2}\right] } & =h-e_{3}-e_{4}-e_{9}-e_{10}, \\
{\left[u_{4,1}\right] } & =h-e_{1}-e_{5}-e_{8}-e_{10} .
\end{aligned}
$$

Proof In Lemma 4.2, we showed that there is an elliptic fibration with two $I_{2}$ fibers, two $I_{4}$ fibers, and a section, and we specified the homology classes of the spheres making up the singular fibers and the section. According to that notation, the spheres 
in the two $I_{2}$ fibers were $\widetilde{C}_{1}, \widetilde{L}_{1}$ and $\widetilde{C}_{2}, \widetilde{L}_{2}$, respectively. The spheres in the $I_{4}$ fibers were denoted $\tilde{X}_{3}, \tilde{Y}_{3}, \widetilde{Z}_{3}, \widetilde{E}_{3}$, and $\tilde{X}_{4}, \tilde{Y}_{4}, \widetilde{Z}_{4}, \widetilde{E}_{4}$, respectively. $\widetilde{C}_{1}, \widetilde{C}_{2}$, $\widetilde{Z}_{3}$ and $\widetilde{E}_{4}$ intersected the section $E_{2}$.

To construct the embedding of $\mathcal{Q}$, first blow up at the intersection of $\tilde{Y}_{4}$ and $\tilde{Z}_{4}$ to produce two adjacent -3 -spheres. Then we can perturb the fibration near one of the $I_{2}$ fibers to split it into two fishtail fibers. Blow up each the self-intersection points of each of these fishtail fibers to create two $-4-$ spheres. To produce the central -5-sphere, take the symplectic resolution of the section $E_{2}$, the two blown-up fishtail fibers, and $E_{4}$ (the sphere in the blown-up $I_{4}$ fiber which intersects the section). The remaining spheres can be taken to be $u_{1,1}=\widetilde{C}_{2}, u_{2,1}=\widetilde{Z}_{3}, u_{2,2}=\tilde{Y}_{3}, u_{3,1}=\tilde{X}_{4}$, $u_{3,2}=\bar{Z}_{4}, u_{4,1}=\bar{Y}_{4}$, where $\bar{C}$ denotes the proper transform under blow-up. Using the homology computation indicated in Lemma 4.2, the homology classes for the $u_{i, j}$ follows.

Lemma 5.16 The element $R \in H_{2}\left(\mathbb{C P}^{2} \# 12 \overline{\mathbb{C P}^{2}}\right)$ defined by

$$
\begin{aligned}
R=533 h-188 e_{1}-186 e_{2}-192 e_{3}-126 e_{4}-185 e_{5}-189 e_{6} \\
-156 e_{7}-104 e_{8}-159 e_{9}-56 e_{10}-0 e_{11}-151 e_{12}
\end{aligned}
$$

satisfies the following conditions:

(1) $R \cdot\left[u_{i, j}\right]=0$ for all $i$ and $j$,

(2) $R \cdot R>0$,

(3) $R \cdot h>0$,

(4) $R \cdot K>0$, where $K=-3 h+e_{1}+\cdots+e_{12}$ is the canonical class of $\mathbb{C P}^{2} \# 12 \overline{\mathbb{C P}^{2}}$.

Proof All of the above claims can be verified by a direct computation.

Proposition 5.17 The result of star surgery on this embedding of $\mathcal{Q}$ into $\mathbb{C P}^{2} \# 12 \overline{\mathbb{C P}^{2}}$ is an exotic copy of $\mathbb{C P}^{2} \# 7 \overline{\mathbb{C P}^{2}}$ which supports a symplectic structure.

Proof Let $X^{\prime}=\left(\left(\mathbb{C P} P^{2} \# 12 \overline{\mathbb{C P}^{2}}\right) \backslash \mathcal{Q}\right) \cup \mathcal{R}$ be the result of star surgery. Then $\chi\left(X^{\prime}\right)=$ $\chi\left(\mathbb{C P}^{2} \# 12 \overline{\mathbb{C P}^{2}}\right)-\chi(\mathcal{Q})+\chi(\mathcal{R})=15-8+3=10$. Since $\mathcal{Q}$ and $\mathcal{R}$ are both negative-definite, $\sigma\left(X^{\prime}\right)=-6$. Since $\mathcal{R}$ is simply connected, $X^{\prime}$ is simply connected. Therefore $X^{\prime}$ is homeomorphic to $\mathbb{C} \mathrm{P}^{2} \# 7 \overline{\mathbb{C} P^{2}}$ by Freedman's theorem [19]. Recall that for 4-manifolds with $b_{2}^{+}=1$, the Seiberg-Witten invariants depend upon a choice of a chamber. Let $h$ denote the homology class of $\mathbb{C} P^{1} \subset \mathbb{C P}^{2} \# 12 \overline{\mathbb{C P}^{2}}$. This class gives the chamber of positive scalar curvature metric, so $\mathrm{SW}_{\mathbb{C P}^{2} \# 12 \overline{\mathbb{C P}^{2}}, h}(L)=0$ for every characteristic class $L$. In particular for the canonical class $K$, we have 


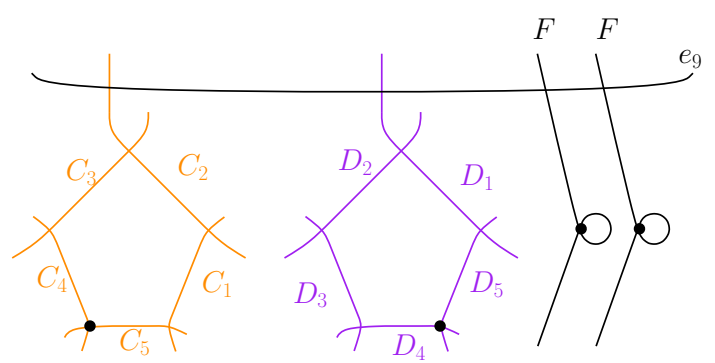

Figure 18: Elliptic fibration on $\mathrm{E}(1)$ with section.

$\mathrm{SW}_{\mathbb{C P}^{2} \# 12 \overline{\mathbb{C P}^{2}}, h}(K)=0$. By Lemma 5.16, the class $R$ determines a chamber and there is a wall between this chamber and the chamber of $h$ with respect to $K$. Hence

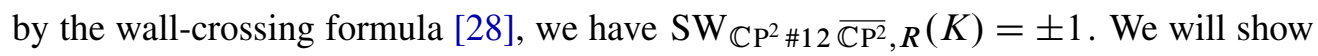
that $X^{\prime}$ also has a nonzero Seiberg-Witten invariant. Indeed the 3 -manifold $\partial S$ is a monopole $L$-space. Let $K_{X^{\prime}}$ denote the canonical class of $X^{\prime}$. Clearly we have

$$
\left.K_{X^{\prime}}\right|_{X^{\prime} \backslash \mathcal{R}}=\left.K\right|_{\mathbb{C P}^{2} \# 12 \overline{\mathbb{C P}^{2}} \backslash \mathcal{Q}} \quad \text { and } \quad d_{X^{\prime}}\left(K_{X^{\prime}}\right)=d_{\mathbb{C P}^{2} \# 12 \overline{\mathbb{C P}^{2}}}(K)=0 .
$$

Hence by [30], we have

$$
\mathrm{SW}_{X^{\prime}, R}\left(K_{X^{\prime}}\right)=\mathrm{SW}_{\mathbb{C P}^{2} \# 12 \overline{\mathbb{C P}^{2}}, R}(K)= \pm 1 .
$$

For manifolds with $b_{2}^{-} \leq 9$, the choice of a chamber is unique. Therefore $X^{\prime}$ is not diffeomorphic to $\mathbb{C} P^{2} \# 7 \overline{\mathbb{C} P^{2}}$ whose Seiberg-Witten invariants are all zero in the unique chamber of positive scalar curvature.

\section{C An exotic $\mathbb{C P}^{2} \# \overline{6} \overline{\mathbb{C} P^{2}}$}

For this construction we use the elliptic fibration with two $I_{5}$ fibers, two fishtails, and a section, with homology classes specified in Lemma 4.3

Lemma 5.18 The configuration of spheres $\mathcal{U}$ embeds into $\mathbb{C} \mathrm{P}^{2} \# 13 \overline{\mathbb{C}} \mathrm{P}^{2}$.

Proof By blowing up at one singular point in each $I_{5}$ fiber, we obtain four -3 -spheres which will make up the ends of each of the arms in the configuration $\mathcal{U}$. The central sphere of square -5 is obtained by taking the symplectic resolution of a section with two blown-up fishtail fibers as well as the -2 -spheres in each $I_{5}$ fiber which intersect this section. 


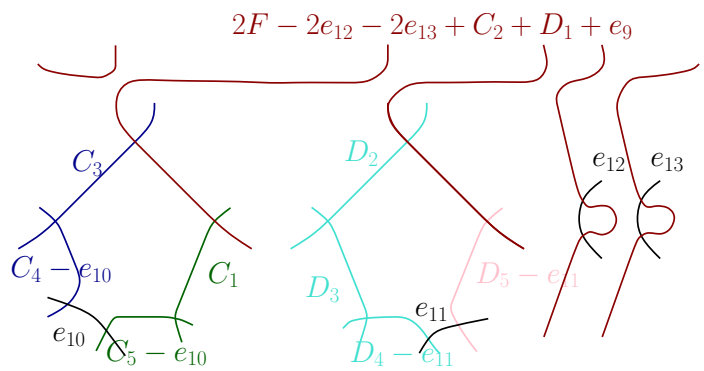

Figure 19: Embedding of $\mathcal{U}$ into $\mathbb{C P}^{2} \# 13 \overline{\mathbb{C P}^{2}}$.

The configuration is shown in Figure 19, and the homology classes are given below:

$$
\begin{aligned}
& {\left[u_{0}\right]=2[F]-2 e_{12}-2 e_{13}+\left[C_{2}\right]+\left[D_{1}\right]+e_{9},} \\
& {\left[u_{1,1}\right]=\left[D_{2}\right], \quad\left[u_{2,1}\right]=\left[C_{3}\right], \quad\left[u_{3,1}\right]=\left[C_{1}\right], \quad\left[u_{4,1}\right]=\left[D_{5}\right]-e_{11} .} \\
& {\left[u_{1,2}\right]=\left[D_{3}\right], \quad\left[u_{2,2}\right]=\left[C_{4}\right]-e_{10}, \quad\left[u_{3,2}\right]=\left[C_{5}\right]-e_{10},} \\
& {\left[u_{1,3}\right]=\left[D_{4}\right]-e_{11},}
\end{aligned}
$$

Lemma 5.19 The element $R \in H_{2}\left(\mathbb{C} P^{2} \# 13 \overline{\mathbb{C P}^{2}}\right)$ defined by

$$
\begin{array}{r}
R=5656 h-1728 e_{1}-1846 e_{2}-1836 e_{3}-1915 e_{4}-1905 e_{5}-1728 e_{6}-1600 e_{7} \\
-1905 e_{8}-1890 e_{9}-246 e_{10}-295 e_{11}-393 e_{12}-1241 e_{13}
\end{array}
$$

satisfies the following conditions:

(1) $R \cdot\left[u_{i, j}\right]=0$ for all $i$ and $j$ (ie $R$ is orthogonal to each embedded sphere of $\mathcal{U})$

(2) $R \cdot R>0$,

(3) $R \cdot h>0$,

(4) $R \cdot K>0$, where $K=-3 h+e_{1}+\cdots+e_{13}$ is the canonical class of $\mathbb{C P}^{2} \# 13 \overline{\mathbb{C P}^{2}}$.

Proof The proof is a direct check.

Proposition 5.20 The result of the $(\mathcal{U}, \mathcal{V})$ star surgery on this embedding of $\mathcal{U}$ into $\mathbb{C P}^{2} \# 12 \overline{\mathbb{C P}^{2}}$ is an exotic copy of $\mathbb{C P}^{2} \# 6 \overline{\mathbb{C P}^{2}}$ which supports a symplectic structure.

Proof The proof is similar to the proof of Proposition 5.17. 


\section{D Another symplectic exotic $\mathbb{C} \mathbf{P}^{2} \# \mathbf{\overline { \mathbb { C } }} \overline{\mathbf{P}^{2}}$}

Proposition 5.21 It is possible to symplectically embedded the configuration $\mathcal{K}$ into $\mathbb{C P}^{2} \# 12 \overline{\mathbb{C P}^{2}}$ so that the homology classes of the central sphere $C_{0}$ and the four spheres in the arms $C_{1}, C_{2}, C_{3}, C_{4}$ are given as follows:

$$
\begin{aligned}
& {\left[C_{0}\right]=2 f+e_{1}-2 e_{10}-2 e_{11}-e_{12},} \\
& {\left[C_{1}\right]=h-e_{1}-e_{2}-e_{3},} \\
& {\left[C_{2}\right]=h-e_{1}-e_{4}-e_{5},} \\
& {\left[C_{3}\right]=h-e_{1}-e_{6}-e_{7},} \\
& {\left[C_{4}\right]=h-e_{1}-e_{8}-e_{9},}
\end{aligned}
$$

where $f=3 h-\left(e_{1}+\cdots+e_{9}\right)$.

Proof This proposition follows immediately from blowing up the embedding of $\mathcal{S}_{2}$ into $\mathbb{C P}^{2} \# 11 \overline{\mathbb{C P}^{2}}$ given in Lemma 5.1 along a single point on the section.

Note the other elliptic fibrations constructed in this paper can be used to find other embeddings of $\mathcal{S}_{2}$ and $\mathcal{K}$ into blow-ups of $E(1)$. It is an interesting question to ask whether the same star surgery operation performed on different embeddings of the plumbing into the same manifold can result in nondiffeomorphic manifolds.

Theorem 5.22 The manifold resulting from star surgery on this embedding of $\mathcal{K}$ into $\mathbb{C P}^{2} \# 12 \overline{\mathbb{C P}^{2}}$ is an exotic copy of $\mathbb{C P}^{2} \# 8 \overline{\mathbb{C P}^{2}}$.

Proof The manifold is homeomorphic to $\mathbb{C P}^{2} \# 8 \overline{\mathbb{C P}^{2}}$ by Freedman's theorem once we show that it is simply connected and has Euler characteristic 11 and signature -7 . The Euler characteristic and signature computations follow from the fact that $\chi(\mathcal{L})=2$, $\sigma(\mathcal{L})=-1, \chi(\mathcal{K})=6$, and $\sigma(\mathcal{K})=-5$. The manifold is simply connected as in the proof of Lemma 5.2 because the generator of $\pi_{1}(\mathcal{L})$ is isotopic to the meridian of $C_{4}$ in the embedding which is homotopically trivial because $C_{4}$ is one sphere in an $I_{3}$-fiber where the other transversally intersecting spheres are not cut out in the star surgery.

The diffeomorphism type can be distinguished from $\mathbb{C P}^{2} \# 8 \overline{\mathbb{C P}^{2}}$ either by showing that the resulting symplectic manifold has Kodaira dimension two as in Lemma 5.5, or by showing that the canonical class and its negation are basic classes in the small perturbation chamber of the star surgered manifold as in Lemma 5.12. In fact the value of $K_{X} \cdot \omega_{X}$ to compute the Kodaira dimension comes out to be exactly the same value as for the star surgery using $\mathcal{S}_{2}$ and $\mathcal{T}_{2}$, and the element $V$ defining the chamber on $\mathbb{C P}^{2} \# 12 \overline{\mathbb{C P}^{2}}$ which descends to the small perturbation chamber in the star surgered manifold can be defined identically as in Lemma 5.11. 
Similar constructions could yield applications of the $(\mathcal{M}, \mathcal{N})$ and $(\mathcal{O}, \mathcal{P})$ star surgeries, using the embeddings yielding the exotic copies of $\mathbb{C P}^{2} \# 7 \overline{\mathbb{C P}^{2}}$ and $\mathbb{C P}^{2} \# 5 \overline{\mathbb{C P}^{2}}$ of sections $5 \mathrm{~B}$ and $5 \mathrm{C}$.

\section{Infinitely many exotic smooth structures on $\mathbb{C} \mathbf{P}^{2} \# 7 \overline{\mathbb{C} \mathbf{P}^{2}}$}

Using a different embedding of $\mathcal{S}_{2}$, we can produce other examples of exotic 4manifolds using star surgery. Here we find an embedding which produces an exotic $\mathbb{C P}^{2} \# 7 \overline{\mathbb{C}} \mathrm{P}^{2}$ by combining our star surgery operation with a knot surgery in the double node neighborhood which was introduced by Fintushel and Stern [16]. Thanks to the knot surgery we will have a better control over the Seiberg-Witten invariants of our manifold. The price we pay is that our manifold is no longer symplectic.

Proof of Theorem 1.3 We will start with the elliptic fibration with two $I_{2}$ fibers, and two $I_{4}$ fibers whose homology classes are specified in Lemma 4.2. The homology classes of these spheres in $\mathbb{C P}^{2} \# k \overline{\mathbb{C} P^{2}}$ will be crucial to our computation of the SeibergWitten invariant of the manifold resulting from star surgery along this embedding.

We follow the same steps as in [16]. Let $K_{n}$ denote the $n$-twist knot. Recall that $K_{n}$ admits a Seifert surface of genus one and its symmetrized Alexander polynomial is given by

$$
\Delta_{K_{n}}(t)=n t-(2 n+1)+n t^{-1} .
$$

Consider the elliptic fibration described in Lemma 4.2. We pick one of the $I_{2}$ fibers, say the one consisting of $\widetilde{C}_{1}$ and $\widetilde{L}_{1}$, and perturb the fibration locally so the $I_{2}$ fiber turns into a double node neighborhood. Then we pick a regular fiber in the double node neighborhood and do knot surgery on it. The knot surgery operation does not preserve the fibration structure inside the double node neighborhood. In particular the section represented by the exceptional sphere $E_{2}$ does not survive after the knot surgery. On the other hand if we choose the gluing map in the knot surgery carefully, then $E_{2}$ turns into a pseudosection, an immersed sphere with one transverse self-intersection which is a section outside of the double node neighborhood.

Before describing our construction further, we would like to make a couple of observations about the manifold $E(1)_{K_{n}}$ which is the result of the knot surgery described above. First note that $E(1)_{K_{n}}$ is simply connected: the fundamental group of the complement of a regular fiber is generated by a normal circle which bounds a disk in $E_{2}$. The second observation is that the only Seiberg-Witten basic classes of $E(1)_{K_{n}}$ are $\pm \operatorname{PD}([F])$, where $[F]$ is the fiber class, and the small perturbation Seiberg-Witten 


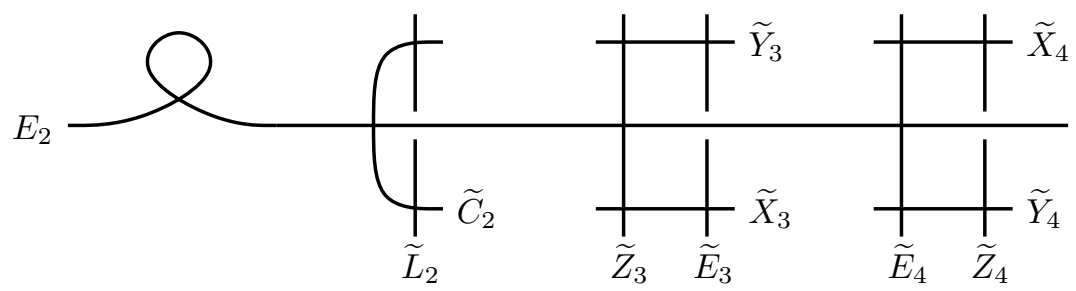

Figure 20: $E(1)_{K_{n}}$.

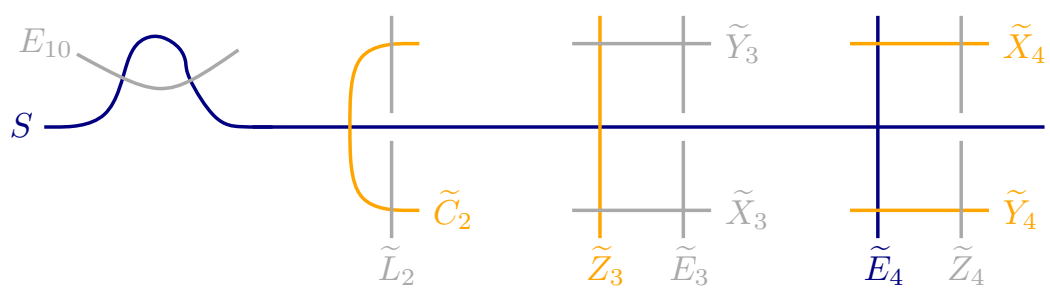

Figure 21: Configuration $\mathcal{S}_{2}$ inside $E(1)_{K_{n}} \# \overline{\mathbb{C P}^{2}}$.

invariants at these classes are $n[46 ; 15]$. Hence $E(1)_{K_{n}}$ is homeomorphic but not diffeomorphic to $E(1)=\mathbb{C P} \mathrm{P}^{2} \# 9 \overline{\mathbb{C P}^{2}}$.

We continue with the construction. Blow up $E(1)_{K_{n}}$ at the double point of the pseudosection $E_{2}$. The proper transform of the pseudo-section is an embedded sphere $S$ whose homology class is represented by $e_{2}-2 e_{10}$. Now we see the configuration $\mathcal{S}_{2}$ embedded in $E(1)_{K_{n}} \# \overline{\mathbb{C P}^{2}}$ using Lemma 4.2:

$$
\begin{aligned}
& {\left[u_{0}\right]=[S]+\left[\widetilde{E}_{4}\right]=e_{1}-2 e_{10},} \\
& {\left[u_{1}\right]=\left[\widetilde{C}_{2}\right]=2 h-e_{1}-e_{2}-e_{3}-e_{4}-e_{5}-e_{6},} \\
& {\left[u_{2}\right]=\left[\widetilde{Z}_{3}\right]=h-e_{1}-e_{2}-e_{9},} \\
& {\left[u_{3}\right]=\left[\tilde{X}_{4}\right]=h-e_{1}-e_{6}-e_{7},} \\
& {\left[u_{4}\right]=\left[\tilde{Y}_{4}\right]=h-e_{1}-e_{5}-e_{8} .}
\end{aligned}
$$

Let $Y_{n}$ denote the result of star surgery of $E(1)_{K_{n}} \# \overline{\mathbb{C P}^{2}}$ along the configuration $\mathcal{S}_{2}$ described above. We claim that $Y_{n}$ is homeomorphic to $\mathbb{C P}^{2} \# 7 \overline{\mathbb{C P}^{2}}$. First we must see that $Y_{n}$ is simply connected. By Proposition 3.1, the generator of $\pi_{1}\left(\mathcal{T}_{2}\right)$ is represented by the curve in $\partial \mathcal{T}_{2}=\partial \mathcal{S}_{2}$ given by the boundary of the normal disk to any of the -2 -spheres in $\mathcal{S}_{2}$. We will take the representative curve which bounds the normal disk to $u_{2}$. Since $u_{2}$ is one -2 -sphere in the $I_{4}$ fiber, and there are other -2 -spheres in that fiber which intersect $u_{2}$ transversally but are otherwise disjoint from the embedding of $\mathcal{S}_{2}\left(\tilde{X}_{3}\right.$ or $\left.\tilde{E}_{3}\right)$, this curve bounds a disk in $E(1)_{K_{n}} \# \overline{\mathbb{C P}} \bar{P}^{2} \backslash \mathcal{S}_{2}=Y_{n} \backslash \mathcal{T}_{2}$. Therefore $Y_{n}$ 
is simply connected. Next we check that $Y_{n}$ has the same Euler characteristic, signature, and parity of $\mathbb{C P}^{2} \# 7 \overline{\mathbb{C P}^{2}}$. A simple computation shows

$$
\chi\left(Y_{n}\right)=\chi\left(E(1)_{K_{n}} \# \overline{\mathbb{C P}^{2}}\right)-\chi\left(\mathcal{S}_{2}\right)+\chi\left(\mathcal{T}_{2}\right)=10,
$$

and

$$
\sigma\left(Y_{n}\right)=\sigma\left(E(1)_{K_{n}} \# \overline{\mathbb{C P}}\right)-\sigma\left(\mathcal{S}_{2}\right)+\sigma\left(\mathcal{T}_{2}\right)=-6 .
$$

Since $b_{2}\left(Y_{n}\right)=8$ and $b_{2}^{+}\left(Y_{n}\right)=1$, the intersection form cannot be a direct sum of hyperbolic pieces and $E_{8}$ 's, so the parity of $Y_{n}$ is odd. Therefore $Y_{n}$ is homeomorphic to $\mathbb{C P}^{2} \# 7 \overline{\mathbb{C P}^{2}}$ by Freedman's theorem.

Finally we compute the Seiberg-Witten invariants of $Y_{n}$. By the blow-up formula $E(1)_{K_{n}} \# \overline{\mathbb{C P}^{2}}$ has exactly four Seiberg-Witten basic classes $\pm \operatorname{PD}([F]) \pm e_{10}$. The small perturbation Seiberg-Witten invariant in the chamber of $h$ evaluates as $\pm n$. We need to translate this information to a chamber whose representative homology class is orthogonal $\mathcal{S}_{2}$. Consider the following homology class:

$$
H:=50 h-32 e_{1}-14 e_{2}-12 e_{3}-21 e_{4}-5 e_{5}-15 e_{6}-3 e_{7}-12 e_{8}-4 e_{9}-16 e_{10} .
$$

It can be checked that $H \cdot H>0, H \cdot h>0$, and $H \cdot\left[u_{i}\right]=0$ for all $i=0, \ldots, 4$. Let $K=-\operatorname{PD}([F])-e_{10}=-3 h+e_{1}+\cdots+e_{9}-e_{10}$. We have $K \cdot H<0$ and $K \cdot h<0$. Hence there is no wall between the chambers determined by $H$ and $h$ with respect to $K$. Note that $\left.K\right|_{\mathcal{S}_{2}}$ is the canonical class of $\mathcal{S}_{2}$, so $K$ descends to $Y_{n}$ as a characteristic class $\widetilde{K}$ with $\left.\widetilde{K}\right|_{\mathcal{T}_{2}}=0$.

Let $X_{n}=E(1)_{K_{n}} \# \overline{\mathbb{C P}^{2}}$, we will check if $d_{X_{n}}(K) \geq 0$ and $d_{Y_{n}}(\tilde{K}) \geq 0$. Clearly

$$
d_{X_{n}}(K)=\frac{K^{2}-3 \sigma\left(X_{n}\right)-2 \chi\left(X_{n}\right)}{4}=\frac{-1-3(-9)-2(13)}{4}=0 .
$$

On the other hand

$$
\begin{aligned}
d_{Y_{n}}(\tilde{K}) & =\frac{\tilde{K}^{2}-3 \sigma\left(Y_{n}\right)-2 \chi\left(Y_{n}\right)}{4} \\
& =\frac{(K)^{2}-\left(\left.K\right|_{\mathcal{S}_{2}}\right)^{2}+\left(\left.\tilde{K}\right|_{\mathcal{T}_{2}}\right)^{2}-3 \sigma\left(Y_{n}\right)-2 \chi\left(Y_{n}\right)}{4} \\
& =\frac{(-1)-(-3)+(0)-3(-6)-2(10)}{4} \\
& =0 .
\end{aligned}
$$

Hence by Theorem 5.10, we have

$$
\left|\mathrm{SW}_{Y_{n}, H}( \pm \tilde{K})\right|=\left|\mathrm{SW}_{E(1)_{K n} \# \overline{\mathbb{C P}^{2}}, H}( \pm K)\right|=n .
$$


Since the small perturbation Seiberg-Witten invariant is well-defined for those manifolds with $b_{2}^{-} \leq 9$, we conclude that $Y_{n}$ has at least two basic classes. In particular $Y_{n}$ is not diffeomorphic to $\mathbb{C P}^{2} \# 7 \overline{\mathbb{C P}^{2}}$, which does not have any basic classes.

It remains to prove the minimality of $Y_{n}$ for $n \geq 2$. By the blow-up formula, it suffices to show that there are exactly two basic classes whose Seiberg-Witten invariants are $\pm n$. We will show that $\pm \widetilde{K}$ are the only Seiberg-Witten basic classes of $Y_{n}$ satisfying $\left|\mathrm{SW}_{Y_{n}}( \pm \tilde{K})\right|=n$. In other words, we will prove that the cohomology class $P:=-\mathrm{PD}([F])+e_{10}$ (the only other basic class up to sign of $X_{n}$ ), does not descend to a basic class of $Y_{n}$. Suppose, to the contrary, that there is a basic class $\widetilde{P}$ of $Y_{n}$ such that $\left.\widetilde{P}\right|_{Y_{n}-\mathcal{T}_{2}}=\left.P\right|_{X_{n} \backslash \mathcal{S}_{2}}$. Then

$$
\begin{aligned}
d_{Y_{n}}(\widetilde{P}) & =\frac{\widetilde{P}^{2}-3 \sigma\left(Y_{n}\right)-2 \chi\left(Y_{n}\right)}{4} \\
& =\frac{(P)^{2}-\left(\left.P\right|_{\mathcal{S}_{2}}\right)^{2}+\left(\left.\widetilde{P}\right|_{\mathcal{T}_{2}}\right)^{2}-3 \sigma\left(Y_{n}\right)-2 \chi\left(Y_{n}\right)}{4} \\
& =\frac{(-1)-(-1 / 3)+\left(\left.\widetilde{P}\right|_{\mathcal{T}_{2}}\right)^{2}-3(-6)-2(10)}{4} \\
& =-\frac{13}{6}+\frac{1}{4}\left(\left.\widetilde{P}\right|_{\mathcal{T}_{2}}\right)^{2}<0 .
\end{aligned}
$$

The last inequality follows from the fact that the intersection form of $\mathcal{T}_{2}$ is negativedefinite. This contradicts the assumption that $\widetilde{P}$ is a basic class.

\section{References}

[1] A Akhmedov, Small exotic 4-manifolds, Algebr. Geom. Topol. 8 (2008) 1781-1794 MR2448872

[2] A Akhmedov, R İ Baykur, B D Park, Constructing infinitely many smooth structures on small 4-manifolds, J. Topol. 1 (2008) 409-428 MR2399137

[3] A Akhmedov, B D Park, Exotic smooth structures on small 4-manifolds, Invent. Math. 173 (2008) 209-223 MR2403396

[4] A Akhmedov, B D Park, Exotic smooth structures on small 4-manifolds with odd signatures, Invent. Math. 181 (2010) 577-603 MR2660453

[5] S Baldridge, $\mathbf{P}$ Kirk, A symplectic manifold homeomorphic but not diffeomorphic to $\mathbb{C P}^{2} \# 3 \overline{\mathbb{C P}}^{2}$, Geom. Topol. 12 (2008) 919-940 MR2403801

[6] M Bhupal, B Özbağci, Symplectic fillings of lens spaces as Lefschetz fibrations (2013) arXiv: 1307.6935

[7] M Bhupal, A I Stipsicz, Weighted homogeneous singularities and rational homology disk smoothings, Amer. J. Math. 133 (2011) 1259-1297 MR2843099 
[8] V Colin, P Ghiggini, K Honda, Equivalence of Heegaard Floer homology and embedded contact homology via open book decompositions, Proc. Natl. Acad. Sci. USA 108 (2011) 8100-8105 MR2806645

[9] S K Donaldson, Irrationality and the $h$-cobordism conjecture, J. Differential Geom. 26 (1987) 141-168 MR892034

[10] H Endo, Y Z Gurtas, Lantern relations and rational blowdowns, Proc. Amer. Math. Soc. 138 (2010) 1131-1142 MR2566578

[11] H Endo, T E Mark, J Van Horn-Morris, Monodromy substitutions and rational blowdowns, J. Topol. 4 (2011) 227-253 MR2783383

[12] J B Etnyre, B Özbağci, Invariants of contact structures from open books, Trans. Amer. Math. Soc. 360 (2008) 3133-3151 MR2379791

[13] R Fintushel, B D Park, R J Stern, Reverse engineering small 4-manifolds, Algebr. Geom. Topol. 7 (2007) 2103-2116 MR2366188

[14] R Fintushel, R J Stern, Rational blowdowns of smooth 4-manifolds, J. Differential Geom. 46 (1997) 181-235 MR1484044

[15] R Fintushel, RJ Stern, Knots, links, and 4-manifolds, Invent. Math. 134 (1998) 363-400 MR1650308

[16] R Fintushel, R J Stern, Double node neighborhoods and families of simply connected 4-manifolds with $b^{+}=1$, J. Amer. Math. Soc. 19 (2006) 171-180 MR2169045

[17] R Fintushel, R J Stern, Six lectures on four 4-manifolds, from: "Low dimensional topology”, (T S Mrowka, P S Ozsváth, editors), IAS/Park City Math. Ser. 15, Amer. Math. Soc. (2009) 265-315 MR2503498

[18] R Fintushel, R J Stern, Pinwheels and nullhomologous surgery on 4-manifolds with $b^{+}=1$, Algebr. Geom. Topol. 11 (2011) 1649-1699 MR2821436

[19] M H Freedman, The topology of four-dimensional manifolds, J. Differential Geom. 17 (1982) 357-453 MR679066

[20] R Friedman, J W Morgan, On the diffeomorphism types of certain algebraic surfaces. I, J. Differential Geom. 27 (1988) 297-369 MR925124

[21] D Gay, T E Mark, Convex plumbings and Lefschetz fibrations, J. Symplectic Geom. 11 (2013) 363-375 MR3100798

[22] D T Gay, A I Stipsicz, Symplectic rational blow-down along Seifert fibered 3manifolds, Int. Math. Res. Not. 2007 (2007) Art. ID rnm084, 20 MR2376205

[23] D T Gay, A I Stipsicz, Symplectic surgeries and normal surface singularities, Algebr. Geom. Topol. 9 (2009) 2203-2223 MR2558309

[24] R E Gompf, Handlebody construction of Stein surfaces, Ann. of Math. 148 (1998) 619-693 MR1668563 
[25] D Kotschick, On manifolds homeomorphic to $\mathbf{C P}^{2} \# 8 \overline{\mathbf{C P}}^{2}$, Invent. Math. 95 (1989) 591-600 MR979367

[26] Ç Kutluhan, Y-J Lee, C H Taubes, HF $=$ HM I : Heegaard Floer homology and Seiberg-Witten Floer homology (2010) arXiv: 1007.1979

[27] T-J Li, The Kodaira dimension of symplectic 4-manifolds, from: "Floer homology, gauge theory, and low-dimensional topology”, (D A Ellwood, P S Ozsváth, A I Stipsicz, Z Szabó, editors), Clay Math. Proc. 5, Amer. Math. Soc. (2006) 249-261 MR2249257

[28] T J Li, A Liu, Symplectic structure on ruled surfaces and a generalized adjunction formula, Math. Res. Lett. 2 (1995) 453-471 MR1355707

[29] D McDuff, D Salamon, Introduction to symplectic topology, Oxford Mathematical Monographs, The Clarendon Press Oxford University Press, New York (1995) MR1373431

[30] M Michalogiorgaki, Rational blow-down along Wahl type plumbing trees of spheres, Algebr. Geom. Topol. 7 (2007) 1327-1343 MR2350284

[31] J W Morgan, The Seiberg-Witten equations and applications to the topology of smooth four-manifolds, Mathematical Notes 44, Princeton Univ. Press (1996) MR1367507

[32] P Ozsváth, Z Szabó, The symplectic Thom conjecture, Ann. of Math. 151 (2000) 93-124 MR1745017

[33] P Ozsváth, Z Szabó, Holomorphic triangle invariants and the topology of symplectic four-manifolds, Duke Math. J. 121 (2004) 1-34 MR2031164

[34] H Park, A I Stipsicz, Smoothings of singularities and symplectic surgery (2012) arXiv:1211.6830v1

[35] J Park, Seiberg-Witten invariants of generalised rational blow-downs, Bull. Austral. Math. Soc. 56 (1997) 363-384 MR1490654

[36] J Park, Simply connected symplectic 4-manifolds with $b_{2}^{+}=1$ and $c_{1}^{2}=2$, Invent. Math. 159 (2005) 657-667 MR2125736

[37] J Park, A I Stipsicz, Z Szabó, Exotic smooth structures on $\mathbb{C P}^{2} \# 5 \overline{\mathbb{C P}^{2}}$, Math. Res. Lett. 12 (2005) 701-712 MR2189231

[38] U Persson, Configurations of Kodaira fibers on rational elliptic surfaces, Math. Z. 205 (1990) 1-47 MR1069483

[39] L P Roberts, Rational blow-downs in Heegaard-Floer homology, Commun. Contemp. Math. 10 (2008) 491-522 MR2444846

[40] L Starkston, Comparing star surgery to rational blow-down (2014) arXiv: 1407.3293

[41] L Starkston, Symplectic fillings of Seifert fibered spaces, Trans. Amer. Math. Soc. 367 (2015) 5971-6016 MR3347194 
[42] A I Stipsicz, Z Szabó, An exotic smooth structure on $\mathbb{C P}^{2} \# 6 \overline{\mathbb{C P}^{2}}$, Geom. Topol. 9 (2005) 813-832 MR2140993

[43] A I Stipsicz, Z Szabó, J Wahl, Rational blowdowns and smoothings of surface singularities, J. Topol. 1 (2008) 477-517 MR2399141

[44] M Symington, Symplectic rational blowdowns, J. Differential Geom. 50 (1998) 505518 MR1690738

[45] M Symington, Generalized symplectic rational blowdowns, Algebr. Geom. Topol. 1 (2001) 503-518 MR1852770

[46] Z Szabó, Exotic 4-manifolds with $b_{2}^{+}=1$, Math. Res. Lett. 3 (1996) 731-741 MR1426531

[47] C Wendl, Strongly fillable contact manifolds and J-holomorphic foliations, Duke Math. J. 151 (2010) 337-384 MR2605865

Department of Mathematics, Bogazici University

34342 Istanbul, Turkey

Mathematics Department, Stanford University

450 Serra Mall, Building 380, Stanford, CA 78712-1202, United States

cagri.karakurt@boun.edu.tr, lstarkst@stanford.edu

Received: 8 October $2014 \quad$ Revised: 15 September 2015 
